\title{
1 Centromere evolution in the fungal genus Verticillium
}

2 Michael F Seidl ${ }^{1,2 \#}$, H Martin Kramer ${ }^{2}$, David E Cook $^{2,3}$, Gabriel Lorencini Fiorin ${ }^{2}$, Grardy CM

3 van den Berg ${ }^{2}$, Luigi Faino ${ }^{2,4}$, and Bart PHJ Thomma ${ }^{2,5 \#}$

4

$5 \quad{ }^{1}$ Theoretical Biology \& Bioinformatics, Utrecht University, Utrecht, the Netherlands

$6 \quad{ }^{2}$ Laboratory of Phytopathology, Wageningen University, Wageningen, the Netherlands

$7 \quad{ }^{3}$ Plant Pathology, Kansas State University, Manhattan, United States of America

$8 \quad{ }^{4}$ Environmental Biology Department, Sapienza Università di Roma, Rome, Italy

$9{ }^{5}$ University of Cologne, Institute for Plant Sciences, Cluster of Excellence on Plant Sciences

10 (CEPLAS), 50674 Cologne, Germany

11

12 \#Corresponding authors: m.f.seidl@uu.nl and bart.thomma@wur.nl

13

14 Running title: Centromeres across the Verticillium genus 


\section{ABSTRACT}

16 Centromeres are chromosomal regions that are crucial for chromosome segregation during

17 mitosis and meiosis, and failed centromere formation can contribute to chromosomal anomalies.

18 Despite this conserved function, centromeres differ significantly between and even within

19 species. Thus far, systematic studies into the organization and evolution of fungal centromeres

20 remain scarce. In this study, we identified the centromeres in each of the ten species of the fungal

21 genus Verticillium and characterized their organization and evolution. Chromatin

22 immunoprecipitation of the centromere-specific histone CenH3 (ChIP-seq) and chromatin

23 conformation capture (Hi-C) followed by high-throughput sequencing identified eight conserved,

24 large ( $150 \mathrm{~kb})$, AT-, and repeat-rich regional centromeres that are embedded in heterochromatin

25 in the plant pathogen $V$. dahliae. Using Hi-C, we similarly identified repeat-rich centromeres in

26 the other Verticillium species. Strikingly, a single repetitive element is strongly associated with

27 centromeric regions in some but not all Verticillium species. Extensive chromosomal

28 rearrangements occurred during Verticillium evolution, yet only a minority could be linked to

29 centromeres, suggesting that centromeres played a minor role in chromosomal evolution.

30 Nevertheless, the size and organization of centromeres differ considerably between species, and

31 centromere size was found to correlate with the genome-wide repeat content. Overall, our study

32 highlights the contribution of repetitive elements to the diversity and rapid evolution of

33 centromeres within the fungal genus Verticillium.

\section{IMPORTANCE}

36 The genus Verticillium contains ten species of plant-associated fungi, some of which are

37 notorious pathogens. Verticillium species evolved by frequent chromosomal rearrangements that

38 contribute to genome plasticity. Centromeres are instrumental for separation of chromosomes

39 during mitosis and meiosis, and failed centromere functionality can lead to chromosomal 
40 anomalies. Here, we used a combination of experimental techniques to identify and characterize

41 centromeres in each of the Verticillium species. Intriguingly, we could strongly associate a single

42 repetitive element to the centromeres of some of the Verticillium species. The presence of this

43 element in the centromeres coincides with increased centromere sizes and genome-wide repeat

44 expansions. Collectively, our findings signify a role of repetitive elements in the function,

45 organization and rapid evolution of centromeres in a set of closely related fungal species. 


\section{INTRODUCTION}

47 Centromeres are crucial for reliable chromosome segregation during mitosis and meiosis. During

48 this process, centromeres direct the assembly of the kinetochore, a multi-protein complex that

49 facilitates attachment of spindle microtubules to chromatids (1-3). Failure in formation or

50 maintenance of centromeres can lead to aneuploidy, i.e. changes in the number of chromosomes

51 within a nucleus, and to chromosomal rearrangements (3-5). While these processes have been

52 often associated with disease development (6), they can also provide genetic diversity that is

53 beneficial for adaptation to novel or changing environments $(7,8)$. For example, aneuploidy in

54 the budding yeast Saccharomyces cerevisiae can lead to increased fitness under selective

55 conditions, such as the presence of antifungal drugs $(9,10)$. Thus, centromeric instability can

56 contribute to adaptive genome evolution $(11,12)$.

57 Despite their conserved function, centromeres are among the most rapidly evolving

58 genomic regions $(13,14)$ that are typically defined by their unusual (AT-rich) sequence

59 composition, low gene and high repeat density, and heterochromatic nature (13, 15).

60 Nevertheless, centromeres differ significantly in size, composition, and organization between

61 species $(13,16)$. Centromeres in $S$. cerevisiae are only $\sim 125$ nucleotides long and are bound by a

62 single nucleosome containing the centromere-specific histone 3 variant CenH3 (also called

63 CENP-A or Cse4) (17-20). In contrast to these 'point centromeres', centromeres in many other

64 fungi are more variable and larger, and have thus been referred to as 'regional centromeres' (15).

65 For instance, in the opportunistically pathogenic yeast Candida albicans, the CenH3-bound 3-5

$66 \mathrm{~kb}$ long centromeric DNA regions differ significantly between chromosomes, and rapidly

67 diverged from closely related Candida species (21-23). Centromeres in the basidiomycete yeasts

68 Malassezia are similar in size (3-5 kb) but contain a short AT-rich consensus sequence in multiple

69 Malassezia species (11). In Malassezia, chromosomal rearrangements and karyotype changes are

70 driven by centromeric loss through chromosomal breakage or by inactivation through sequence 
71 diversification (11). Chromosomal rearrangements at centromeres have been similarly observed

72 in the yeast Candida parapsilosis, suggesting that centromeres can be fragile and contribute to

73 karyotype evolution $(11,12)$. CenH3-bound centromeric regions of the basidiomycete yeast

74 Cryptococcus neoformans are relatively large, ranging from 30 to $65 \mathrm{~kb}$, and are rich in Long

75 Terminal Repeat (LTR)-type retrotransposons (16). Centromere sizes differ between

76 Cryptococcus species as those lacking RNAi and DNA methylation have shorter centromeres,

77 associated with the loss of full-length LTR retrotransposons at centromeric regions, suggesting

78 that functional RNAi together with DNA methylation is required for centromere stability (16).

79 In filamentous fungi, centromeres have been most extensively studied in the saprophyte

80 Neurospora crassa (15). In this species, centromeric regions are considerably larger than in yeasts

81 (on average $\sim 200 \mathrm{~kb}$ ), and are characterized by AT-rich sequences that are degenerated remnants

82 of transposable elements and sequence repeats that lack an overall consensus sequence $(15,24$,

83 25). The increased AT-content and the degenerated nature of transposable elements in the

84 genome of $N$. crassa are the result of a process called repeat-induced point mutation (RIP) (15,

85 26). RIP has been linked to the sexual cycle of ascomycetes and targets repetitive sequences by

86 inducing $\mathrm{C}$ to $\mathrm{T}$ mutations, preferably at $\mathrm{CpA}$ di-nucleotides (26). The AT-rich centromeric

87 regions are bound by $\mathrm{CenH} 3$ and enriched in the heterochromatin-specific histone modification

88 histone 3 trimethylation of lysine 9 (H3K9me3) (25). Additionally, H3K9me3 and cytosine

89 methylation occurs at the periphery of the centromeres (25). Alterations in H3K9me3 localization

90 compromise centromeric localization, suggesting that the formation and location of

91 heterochromatin, rather than the DNA sequence itself, is essential for function and localization of

92 centromeres in $N$. crassa $(15,25)$. However, heterochromatin is not a hallmark for centromeres in

93 all filamentous fungi. Centromeres in the fungal wheat pathogen Zymoseptoria tritici are shorter

$94(\sim 10 \mathrm{~kb})$ and AT-poor, and their presence does not correlate with transposable elements nor with

95 heterochromatin-specific histone modifications such as $\mathrm{H} 3 \mathrm{~K} 9 \mathrm{me} 3$ or histone 3 trimethylation of 
96 lysine 27 (H3K27me3) (27). Thus, even though centromeric function is highly conserved, fungal

97 centromeres differ considerably in size, sequence composition, and organization.

98 Knowledge on centromeres has been impaired by their repetitive nature, which hampers

99 their assembly and subsequent analyses (15, 28). However, recent advances in long-read

100 sequencing technologies enables to study the constitution and evolution of centromeres (11, 16,

101 29-31). By using long-read sequencing technologies in combination with optical mapping, we

102 previously generated gapless genome assemblies of two strains of the fungal plant pathogen

103 Verticillium dahliae (32), whose genomes are characterized by genome rearrangements and the

104 occurrence of lineage-specific (LS) regions $(7,8,33-35)$ that are hypervariable between $V$.

105 dahliae strains and contain genes with roles in adaptive evolution to plant hosts $(7,8,33,35)$.

106 Repetitive elements within the LS regions display a distinct chromatin state when compared with

107 other repetitive regions (36). The Verticillium genus consists of ten species that are all soil-borne

108 and presumed asexual but have different life-styles (37). Nine of these species are haploid, while

109 the species Verticillium longisporum is an allodiploid hybrid between a strain that is closely

110 related to $V$. dahliae and an unknown Verticillium species (37-39). During the evolution of the

111 different Verticillium species frequent chromosomal rearrangements occurred (8, 35, 40).

112 Facilitated by the availability of high-quality genome assemblies of $V$. dahliae strains and of all

113 other Verticillium species (32, 33, 40, 41), we here sought to identify and study the constitution

114 and evolution of centromeres in the Verticillium genus. 


\section{RESULTS}

\section{CenH3-binding identifies large regional centromeres in Verticillium dahliae}

117 Centromeres differ significantly between fungi, but most centromeres are functionally defined by

118 nucleosomes containing CenH3 (1). To identify centromeres in $V$. dahliae strain JR2 by

119 chromatin immunoprecipitation followed by high-throughput sequencing (ChIP-seq), we first

120 identified the V. dahliae CenH3 ortholog (Fig. S1a) and generated transformants with N-

121 terminally FLAG-tagged CenH3 (Table S1). To this end, the coding sequence for the FLAG-

122 tagged $\mathrm{CenH} 3$ was inserted in locus behind the native CenH3 promotor (Figs. S1b-c). We

123 subsequently used anti-FLAG antibodies to purify FLAG-tagged CenH3-containing nucleosomes

124 from two $V$. dahliae transformants (Table S1a) and sequenced the nucleosome-associated

125 genomic DNA. Mapping of the sequencing reads to the $V$. dahliae strain JR2 genome assembly

126 identified a single CenH3-enriched region per chromosome (Fig. 1a; Fig. S1d-e), while mapping

127 of the sequencing reads derived from the WT strain did not reveal any CenH3-enriched region

128 (Fig. S1d-e). The CenH3-enriched regions, designated as Cen1-8, range between $\sim 94$ and 187

$129 \mathrm{~kb}$ in size (Fig. 1a; Table 1). To corroborate these centromere sizes, we assessed centromere

130 locations based on a previously generated optical map $(32,35)$ revealing no significant size

131 differences (Fig. S1f). Thus, we conclude that CenH3-binding defines large regional centromeres

132 in V. dahliae strain JR2. 


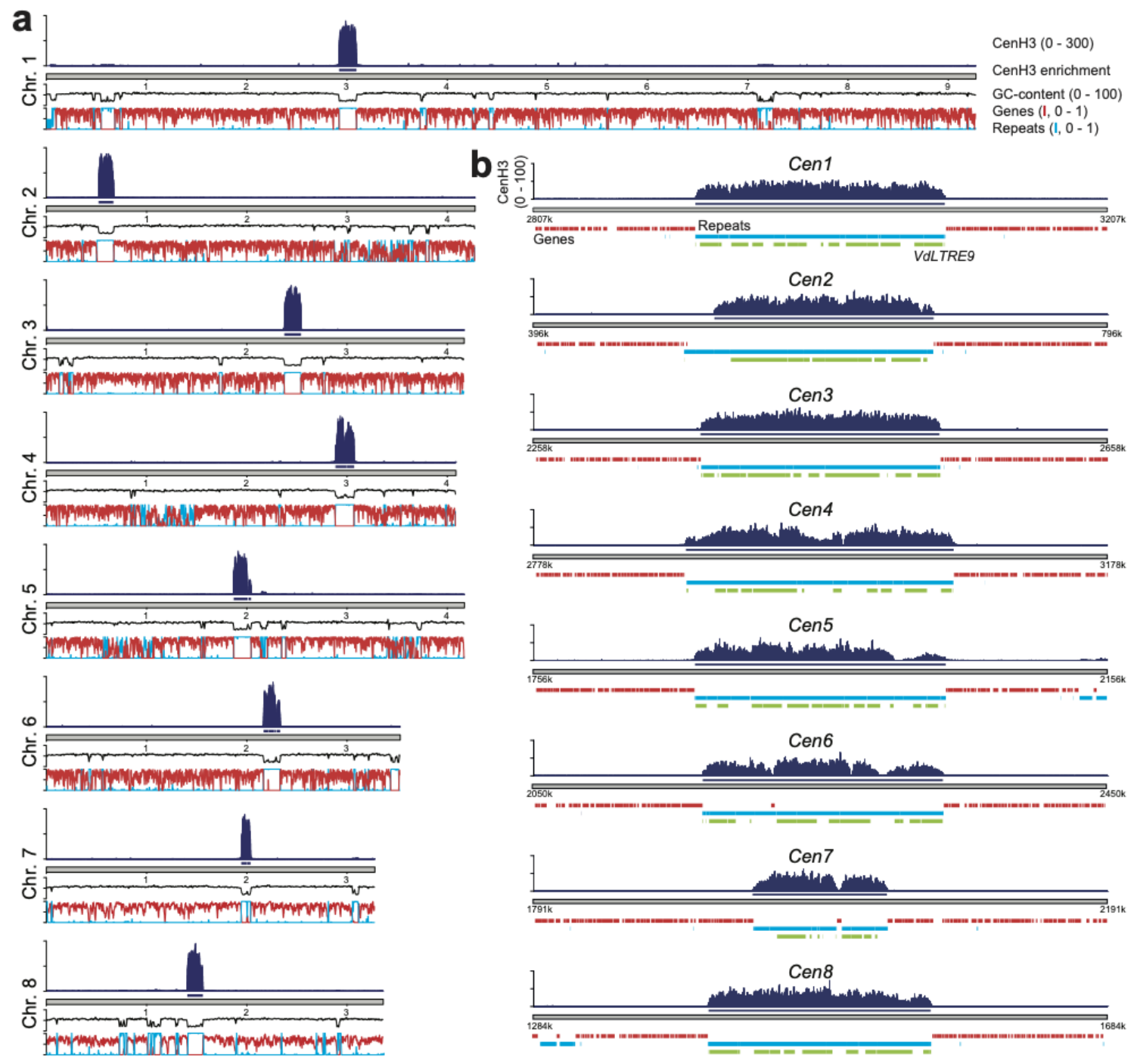

134

Figure 1 - CenH3-binding defines centromeres in Verticillium dahliae strain JR2. (a) Schematic overview of the chromosomes of $V$. dahliae strain JR2 showing the normalized CenH3 ChIP-seq read coverage (RPGC normalization in $1 \mathrm{~kb}$ bins with $3 \mathrm{~kb}$ smoothening), CenH3 enriched regions, GC-content, gene density (red line), and repeat density (blue line). (b) Magnification of a $400 \mathrm{~kb}$ region containing the centromere is shown for each of the eight chromosomes of $V$. dahliae strain JR2 (Cenl-8) depicting the CenH3 ChIP-seq read coverage (RPGC normalization in 10 bp bins with a $30 \mathrm{bp}$ smoothening) and enrichment, as well as the presence of genes (red) and repetitive elements (blue). Regions carrying the centromere-specific long-terminal repeat element VdLTRE9 are highlighted in green. 


\section{Centromeres in Verticillium dahliae are repeat-rich and embedded in heterochromatin}

148 Centromeres are often characterized by increased AT-content, increased repeat density, and

149 depletion of protein coding genes $(13,15,29)$. To characterize the centromeres in $V$. dahliae

150 strain JR2, we queried the eight chromosomes for the presence of large AT-rich, gene-sparse, and

151 repeat-rich regions. Seven of the eight chromosomes contain only a single large ( $>93 \mathrm{~kb}$; average

152 size $\sim 150 \mathrm{~kb}$ ) AT-rich region ( 74-78\% versus $\sim 46 \%$ genome-wide), nearly completely devoid of

153 protein-coding genes and enriched for repetitive sequences, that overlaps with the regions defined

154 by CenH3-binding (Fig. 1a; Table 1). In contrast, chromosome 1 contains three regions with

155 these characteristics (Fig. 1a; Table 1). However, only one of these overlaps with the centromeric

156 regions defined by CenH3-binding (Fig. 1).

157 Elevated AT-levels in repeat-rich regions are caused by RIP mutations in some 158 filamentous fungi $(15,25,26,42)$. Due to its presumably asexual nature (7), the occurrence of 159 RIP in $V$. dahliae is controversial $(8,43,44)$, although signatures of RIP have previously been 160 reported in a subset of repeat-rich regions (36). We assessed the occurrence of RIP signatures in 161 centromeres using the composite RIP index (CRI) (45), which considers C to T mutations in the

162 CpA context. Intriguingly, genomic regions located at centromeres display significantly higher

163 CRI values than other genomic regions (e.g. genes or repetitive elements) (Fig. 2a; Figs. S2, 164 S3a), and thus RIP signatures at repetitive elements located at centromeres likely contribute to the 165 high AT-levels.

166 In most filamentous fungi and oomycetes, AT- and repeat-rich centromeres are embedded

167 in heterochromatin that is characterized by methylated DNA and by particular histone 168 modifications (H3K9me3 and $\mathrm{H} 3 \mathrm{~K} 27 \mathrm{me} 3)(13,15,16,25,30,45)$. We recently determined 169 chromatin states in the genome of $V$. dahliae strain JR2 and revealed that repetitive sequences 170 outside of the LS regions display characteristics of heterochromatin (36). To define centromeric 171 chromatin states, we used previously generated bisulfite sequencing data to monitor DNA 
172 methylation (mC) and ChIP-seq data to determine the distribution of the heterochromatic marks

$173 \mathrm{H} 3 \mathrm{~K} 9 \mathrm{me} 3$ and $\mathrm{H} 3 \mathrm{~K} 27 \mathrm{me} 3$ (36). To also determine the distribution of euchromatin, we performed

174 ChIP-seq with an antibody against the euchromatic mark di-methylation of lysine 4 of histone H3

175 (H3K4me2). We observed overall low genome-wide DNA methylation levels (36) (Fig. 2a; Fig.

176 S2), similar to the previously reported levels for Aspergillus flavus (46) and lower than for $N$.

177 crassa (47). Nevertheless, repetitive elements and centromeres show significantly higher DNA

178 methylation levels in all contexts when compared with genes (Fig. 2b). Methylation (in CG

179 context) at repetitive elements at centromeres is significantly higher than at repeats located along

180 the chromosomal arm, but not at sub-telomeric regions (Fig. 2c), and more methylation at

181 centromeres correlates with increased CRI (Fig. 2a; Figs. S2, S3a). DNA methylation co-

182 localizes with $\mathrm{H} 3 \mathrm{~K} 9 \mathrm{me} 3$ at repeat-rich regions (36) (Figs. 2a; Fig. S2). H3K9me3 occurs

183 predominantly at repetitive elements localized at sub-telomeres and centromeres (Figs. 2d-e;

184 Figs. S2, S3b). In comparison, H3K4me2 and H3K27me3 are largely absent from centromeres

185 (Figs. 2d-e; Fig. S3b). Collectively, these observations indicate that centromeres of V. dahliae

186 display typical characteristics of constitutive heterochromatin. 


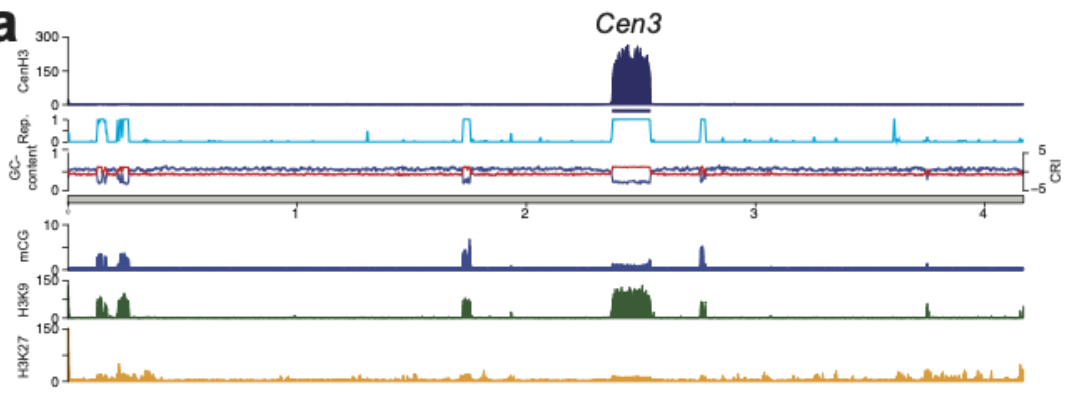

b
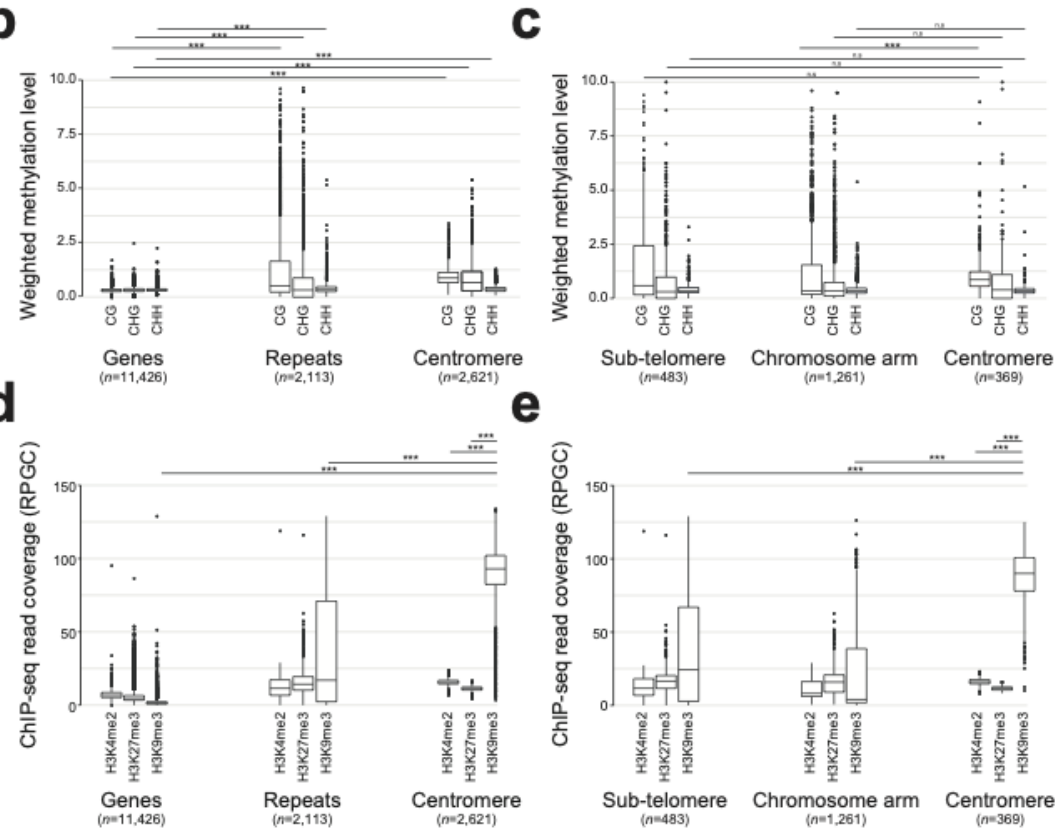

188 Figure 2 - Centromeres in Verticillium dahliae strain JR2 are embedded in heterochromatin. (a) Schematic overview of chromosome 3 of $V$. dahliae strain JR2, exemplifying the distribution of heterochromatin-associated chromatin modifications ( $\mathrm{mC}$, $\mathrm{H} 3 \mathrm{~K} 9 \mathrm{me} 3$, and $\mathrm{H} 3 \mathrm{~K} 27 \mathrm{me} 3$ ) in relation to the centromeres. The different lanes display the CenH3-FLAG ChIP-seq read coverage (RPGC normalization in $1 \mathrm{~kb}$ bins with $3 \mathrm{~kb}$ smoothening), the CenH3-FLAG enriched regions, the repeat-density, the GC-content, the CRI as well as the weighted cytosine methylation (all summarized in $5 \mathrm{~kb}$ windows with $500 \mathrm{bp}$ slide), and the normalized $\mathrm{H} 3 \mathrm{~K} 9 \mathrm{me} 3$ and $\mathrm{H} 3 \mathrm{~K} 27 \mathrm{me} 3 \mathrm{ChIP}$-seq read coverage (RPGC normalization in 1 $\mathrm{kb}$ bins with $3 \mathrm{~kb}$ smoothening). The schematic overview of all chromosomes is shown in Figure S2. (b) Box plots of weighted DNA methylation levels per genomic context (CG, CHG, or CHH) are summarized over genes, repetitive elements, or $5 \mathrm{~kb}$ genomic windows (500 bp slide) overlapping with the centromeric regions. (c) Weighted DNA methylation levels per genomic context ( $\mathrm{CG}, \mathrm{CHG}$, or $\mathrm{CHH})$ are summarized over repetitive elements that have been split based on their genomic location; sub-telomeres (within the first or last $10 \%$ of the chromosome), centromeres, or the remainder of the chromosome arm. (d) ChIP-seq read coverage (RPGC normalized; see (a)) for $\mathrm{H} 3 \mathrm{~K} 4 \mathrm{me} 2, \mathrm{H} 3 \mathrm{~K} 27 \mathrm{~m} 3$, and $\mathrm{H} 3 \mathrm{~K} 9 \mathrm{me} 3$ is summarized over genes, repetitive elements, or $5 \mathrm{~kb}$ windows (500 bp slide) overlapping with the centromeric regions. (e) ChIP-seq read coverage (RPGC normalized; see (a)) for H3K4me2, H3K27m3, and H3K9me3 is summarized over repetitive elements that have been split based on their genomic location; subtelomeres (within the first or last $10 \%$ of the chromosome), centromeres, or the remainder of the 
208 chromosomal arm. Statistical differences for the indicated comparisons were calculated using the one-sided non-parametric Mann-Whitney test; p-values $<0.001$ : ***.

\section{A single repeat associates with Verticillium dahliae strain JR2 centromeres}

212 Centromere identity and function is typically defined by CenH3-binding and not by specific DNA

213 sequences, although various types of repetitive sequences, such as transposable elements, are

214 commonly observed in centromeres of plants, animals, and fungi $(13,15,48,49)$. Unsurprisingly,

215 CenH3-bound centromeres are repeat-rich in V. dahliae (Fig. 1). A detailed analysis of the eight

216 centromeres revealed a near-complete (>96\%) composition of repetitive elements belonging to

217 only ten different repeat sub-families (Figs. 1b, 3a; Table 1), of which the majority shows

218 similarity to LTR retrotransposons of the Gypsy- and Copia-like families (Fig. 3a). These

219 elements show signs of RIP, are highly methylated, and non-transcribed (Figs. S3c-e), and thus

220 likely inactive. Interestingly, a single LTR retrotransposon sub-family, previously designated

$221 \operatorname{VdLTRE9}(8,32)$, covers on average $\sim 70 \%$ of the DNA sequences at the eight centromeres,

222 ranging from $47 \%$ in $C e n 7$ to $83 \%$ in Cen2 (Fig. 3a; Table 1). We scanned the genome for the

223 localization of the ten repeat sub-families (Fig. 3). Intriguingly, although it is one of the most

224 abundant repeats in the genome with 215 complete or partial matches, VdLTR9 is associated to

225 centromeres as $95 \%$ of the copies (204 out of 215; one-sided Fisher's exact test; multiple-testing

226 corrected p-value 3e-106) occur at the eight centromeres, whereas only 5\% of the copies are

227 dispersed over the genome (Fig. 3b-c). The nine other repeat sub-families have additional

228 matches that are located outside of the centromeres (Figs. 1a; Figs. 3b-c), and only two of these

229 repeats are significantly enriched and consistently present in all eight centromeres; 63\% and 45\%

230 of the matches of these two sub-families occur at the centromeres (Fig. 3c). Collectively, these

231 findings suggest that only the presence of VdLTRE9 is strongly associated with centromeres in $V$.

232 dahliae strain JR2. 
234 VdLTRE9 is $\sim 7.3 \mathrm{~kb}$ long (the two LTR sequences are each $200 \mathrm{bp}$ long), and the individual

235 matches share a high degree of sequence identity ( 86\%). Sequence similarity based TE-

236 classifications using PASTEC (50) indicates that the consensus sequence displays remote

237 similarity to Gypsy-like retrotransposons. Only $25 \%$ of the VdLTRE9 matches in the genome

238 cover the entire $(>97.5 \%)$ consensus sequence, but many of these are still fragmented as they

239 occur as discontinuous copies. Furthermore, the VdLTRE9 consensus sequence is AT-rich ( 75\%

240 AT), which may be caused by RIP (Fig. S3d), indicating that VdLTRE9 has significantly

241 degenerated.

a
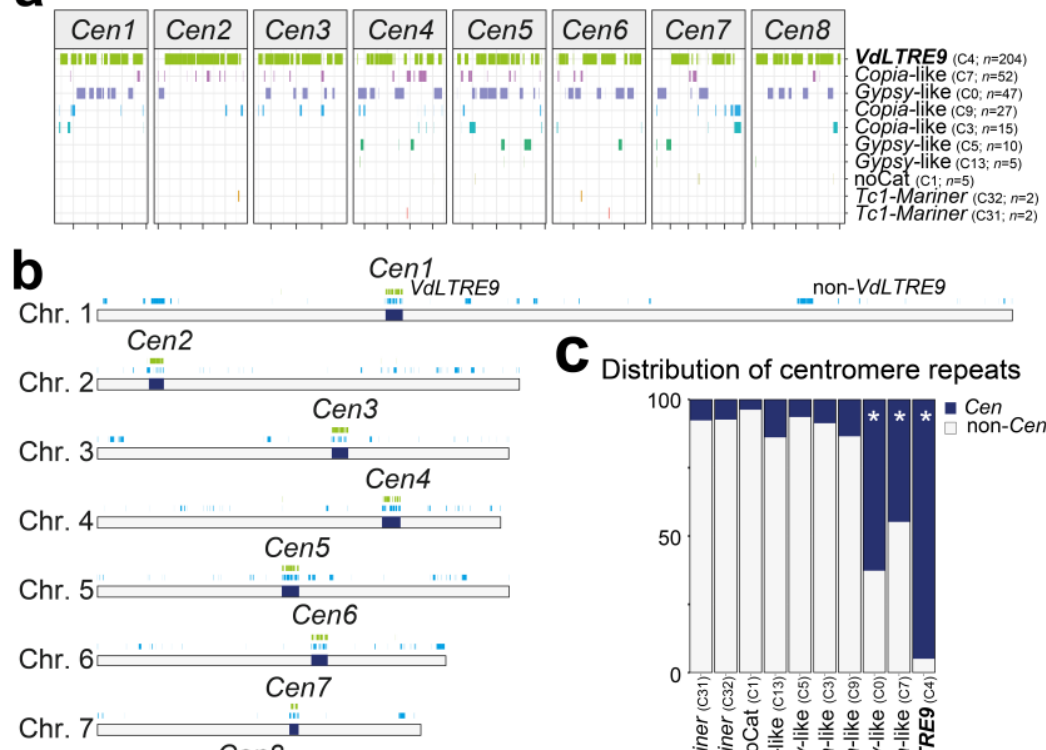

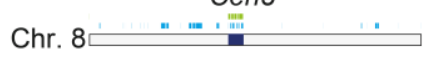

non-VdLTRE9
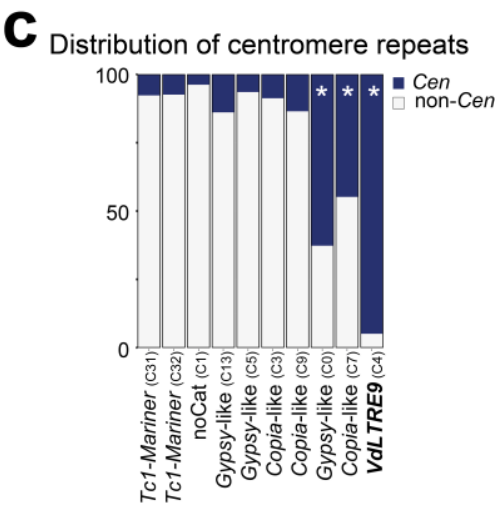

Figure 3 - A single repeat family associates with centromeres in Verticillium dahliae strain JR2. (a) The presence of different repeat sub-families is shown across the eight centromeres (Cen1-8), and the number of occurrences for each sub-family within the centromeres is indicated. The individual centromeres in the diagram are shown in equal scale. (b) Genome-wide distribution of the ten repeat sub-families occurring within the eight centromeres (Cen 1-8; dark blue); the location of VdLTRE9 is shown in green and the location of elements belonging to the other nine sub-repeat families (from panel (a)) is shown in light blue. (c) The distribution of different repeat sub-families in centromeres (Cen; dark blue) and across the genome (non-Cen; light grey). The enrichment of specific sub-families at centromeres was assessed using a onesided Fisher's exact test. Significant enrichment (multiple-testing corrected p-value $<0.01$ ) is denoted with an asterisk. 


\section{VdLTRE9 as hallmark of Verticillium dahliae centromeres}

256 To examine if VdLTRE9 similarly occurs at centromeres in other $V$. dahliae strains, we made use

257 of the complete genome assembly of $V$. dahliae strain VdLs17 $(8,32,35)$. The evolution of $V$.

258 dahliae is characterised by chromosomal rearrangements (8, 35) (Figs. 4a; Figs. S4a-c).

259 Nevertheless, synteny analyses between $V$. dahliae strains JR2 and VdLs17 revealed large regions

260 of co-linearity between chromosomes and identified significant sequence and synteny

261 conservation between the centromeres and their flanking regions (Figs. 4b-c; Fig. S4a),

262 suggesting that centromeric sequences and their locations are conserved. We queried the genome

263 of $V$. dahliae strain VdLs17 for the presence of VdLTRE9 and identified a single region on each

264 chromosome, collectively containing 186 of the 207 (90\%) complete or partial matches of

265 VdLTRE9 in the genome (Fig. 4d) (one-sided Fisher's exact test; multiple-testing corrected p-

266 value 3e-146). These VdLTR9-rich regions are $\sim 150 \mathrm{~kb}$ in size, AT-rich, gene-poor and repeat-

267 rich, and share similarity to the previously identified CenH3-bound and VdLTRE9-enriched

268 regions of $V$. dahliae strain JR2 (Figs. 4b-c; Fig. S4d), suggesting that these regions similarly

269 represent the centromeres of $V$. dahliae strain VdLs17.

270 Centromeres $N$. crassa and some other fungi co-localize within the nucleus $(15,51-55)$. This

271 co-localization can be experimentally determined using chromosome conformation capture (Hi-

272 C), which can identify centromeres by their increased inter-chromosomal contacts (55). To

273 confirm that $\mathrm{Hi}-\mathrm{C}$ can be used to identify centromeres in $V$. dahliae, we first applied Hi-C to $V$.

274 dahliae strain JR2. As anticipated, we observed seven strong inter-chromosomal contacts for each

275 of the eight chromosomes (Figs. 4e). Importantly, the interacting regions overlap with the

276 CenH3-bound regions that we identified as centromeres (Table S1b), demonstrating that

277 centromeres in $V$. dahliae strain JR2 co-localize within the nucleus and supporting that Hi-C

278 reliably identifies centromeres $(51,52)$. We then applied Hi-C to V. dahliae strain VdLs17, and 
279 similarly identified regions with strong inter-chromosomal contacts, one for each of the

280 chromosomes (Figs. 4f). These regions overlap with the VdLTRE9-enriched regions (Table S1b),

281 suggesting that these represent functional centromeres in V. dahliae strain VdLs17.

282 The two V. dahliae strains JR2 and VdLs17 are closely related and differ only by $\sim 0.05 \%$

283 sequence diversity $(8,35)$. Thus, the conservation of VdLTRE9 at centromeres could be driven by

284 limited divergence between the two $V$. dahliae strains rather than representing a hallmark of $V$.

285 dahliae centromeres. Therefore, we sought to determine centromeres in an additional V. dahliae

286 strain with increased sequence diversity when compared with $V$. dahliae strains JR2 or VdLs17,

287 namely strain CQ2 that displays $\sim 1.05$ percent sequence diversity (33). We previously obtained a

288 long-read based genome assembly of this strain that encompasses 17 contigs (33). We generated

289 Hi-C data for V. dahliae strain CQ2 and utilized intra-chromosomal contacts to assign the contigs

290 into eight pseudo-chromosomes, leaving 148 kb unplaced scaffolds (Fig. 4g; Fig. S4e; Table

291 S1c). We subsequently identified a single region with seven strong inter-chromosomal contacts

292 for each pseudo-chromosome that is significantly enriched for VdLTRE9 (one-sided Fisher's

293 exact test; multiple-testing corrected p-value 3.4e-166) (Figs. 4d, g; Fig. S4e; Table S1b).

294 Synteny analyses between $V$. dahliae strains JR2 and CQ2 revealed that the eight VdLTRE9-rich

295 regions and their flanking chromosomal regions are co-linear, suggesting that centromere

296 locations are conserved between different $V$. dahliae strains (Figs. 4; Figs. S4a-c, f). With an

297 average size of $165 \mathrm{~kb}$, the centromeres of $V$. dahliae strain CQ2 are similar in size as the $144 \mathrm{~kb}$

298 and $157 \mathrm{~kb}$ average sizes in $V$. dahliae strains VdLs17 and JR2, respectively (Table S1b). The

299 sizes of the corresponding (i.e. homologous) centromeres vary between the different $V$. dahliae

300 strains. Yet, the consistent co-occurrence of the VdLTRE9-rich regions with the interaction data

301 obtained by Hi-C throughout a selection of $V$. dahliae strains demonstrates that VdLTRE9 is a

302 hallmark of $V$. dahliae centromeres. 
304

305

306

307

308

309

310

311

312

313

314

315

316

317

318

319

320

321

322

323

324

325 b

a Verticillium dahliae

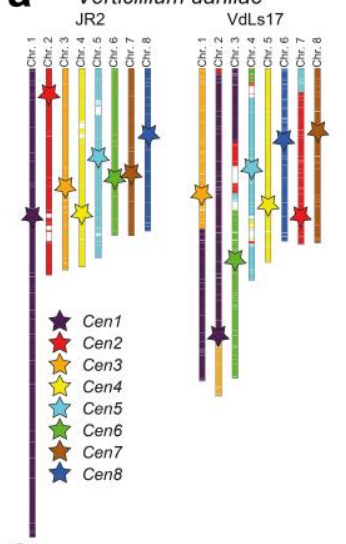

e Verticillium dahliae JR2

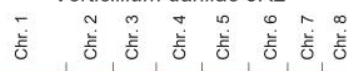
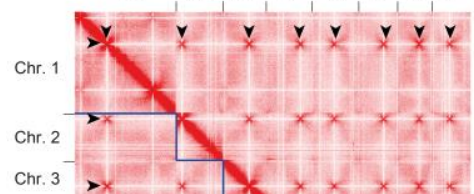

Chr. $3^{-}>$

Chr. 4 ,

Chr. 5 >

Chr. 6 Chr. $7 \rightarrow$

Chr. $\left.{ }^{-}\right]$
Chr. 8

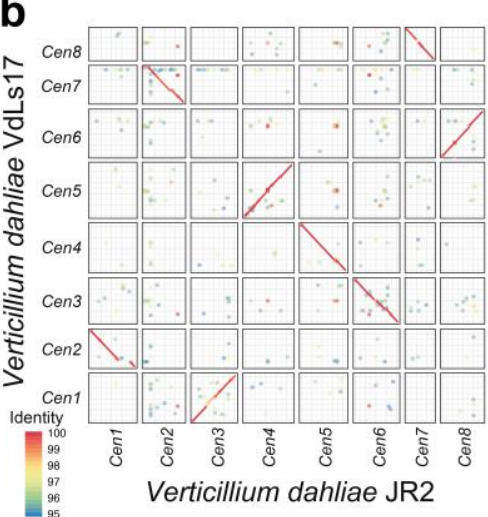

C

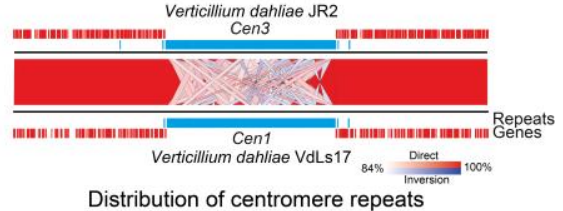

d

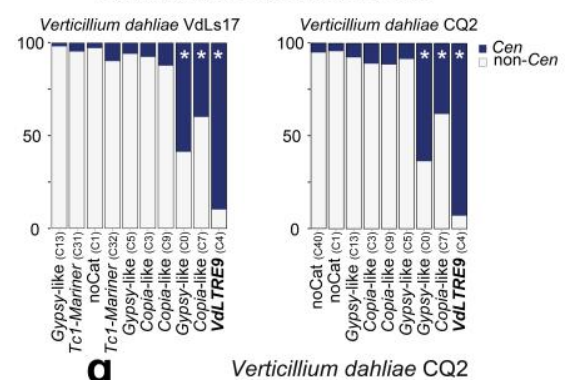

f Verticillium dahliae VdLs17

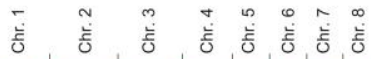

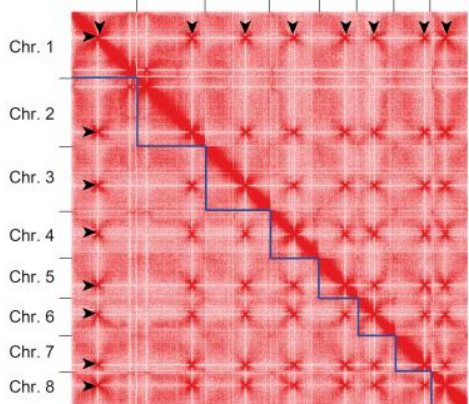

Figure 4 - Hi-C contact maps identify VdLTRE9 as hallmark of centromeres in Verticillium dahliae. (a) Synteny analyses of the eight chromosomes of $V$. dahliae strains JR2 and VdLs17. Schematic overview of the eight chromosomes of $V$. dahliae strain JR2 (left) and the corresponding syntenic regions in $V$. dahliae strains VdLs17 (right). Approximate locations of centromeres are indicated by stars, and syntenic centromeres of $V$. dahliae strain VdLs17 are colored according to Cen1-8 of V. dahliae strain JR2. (b) Sequence alignment of the centromeric regions $\pm 20 \mathrm{~kb}$ in $V$. dahliae strain JR2 and the corresponding regions in $V$. dahliae strains VdLs17 shown as dot-plot. For clarity, only alignments with $>95 \%$ sequence identity are displayed. (c) Magnification of Cen3 of $V$. dahliae strain JR2 and the syntenic Cenl of strain VdLs17. Synteny between regions is indicated by ribbons; entire centromeric regions Cenl and Cen 3 are syntenic and sequence similarity between individual VdLTRE9 elements is visualized. The Cen regions $\pm 150 \mathrm{~kb}$ are shown as well as genes (red) and repeats (blue) are annotated within this region. (d) Distribution of different repeat families in centromeres (Cen; dark blue) and across the genome (non-Cen; light grey) for $V$. dahliae strains VdLs17 and CQ2. The enrichment of specific sub-families at centromeres was assessed using a one-sided Fisher's exact test. Significant enrichment (multiple-testing corrected p-value $<0.01$ ) is denoted with an asterisk. (e-g) Hi-C contact matrix showing interaction frequencies between genomic regions in Verticillium dahliae strains JR2 (e), VdLs17 (f), and CQ2 (g). Regions of high inter-chromosomal interaction frequencies are indicative of centromeres and are highlighted by arrow heads. Interaction frequencies are summarized in $50 \mathrm{~kb}$ bins along the genome. 


\section{The evolution of Verticillium centromeres}

328 In addition to $V$. dahliae, we previously generated genome assemblies of the eight haploid

329 Verticillium species and the allodiploid V. longisporum $(39,40)$ (Fig. 5a) that ranged from 12 to

330684 scaffolds (Table S1c). These ten Verticillium species have been traditionally separated over

331 two distinct clades; Flavnonexudans and Flavexudans (Fig. 5a) (37). We generated Hi-C data to

332 study the composition and evolution of centromeres in the different Verticillium species. By using

333 intra-chromosomal interaction signals, we first assigned the vast majority of the previously

334 assembled contigs into eight pseudo-chromosomes for each of the haploid Verticillium species

335 and 16 pseudo-chromosomes for the diploid $V$. longisporum, leaving between $0.5 \mathrm{~kb}$ and $2,022 \mathrm{~kb}$

336 unassigned (Fig. S5; Table S1c). For most genome assemblies, the pseudo-chromosomes contain

337 one or both telomeric repeats (Table S1c), and thus we conclude that all Verticillium strains have

338 eight chromosomes, and that this number doubled in $V$. longisporum. Based on the inter-

339 chromosomal Hi-C interaction signals, we identified a single region with high inter-chromosomal

340 contacts for each of the pseudo-chromosomes (Fig. S5; Table S1d), indicating that these are the

341 centromeres in the different Verticillium species. The average centromere size in Verticillium is

$342 \sim 80 \mathrm{~kb}$, yet we observed significant differences between the species (Fig. 5b; Figs. S6a-b).

343 Centromeres within the Flavexudans clade are similarly sized and significantly smaller than the

344 genus-wide average. By contrast, $V$. dahliae and $V$. longisporum centromeres are significantly

345 larger.

346 We subsequently assessed whether VdLTRE9 defines centromeres in the other

347 Verticillium species besides $V$. dahliae as well. Interestingly, VdLTRE9 is abundant at

348 centromeres in the allodiploid V. longisporum and in V. alfalfae, but fewer (21) or no VdLTRE9

349 copies were identified at centromeres in V. nonalfalfae and V. nubilum, respectively (Fig. 5c; Fig.

350 S6c-d). Similarly, only few or no (partial) matches of VdLTRE9 consensus could be identified in

351 the genomes of the Flavexudans species (Fig. 5c; Fig. S6-7; Table S1e). Collectively, these 
352 findings demonstrate that VdLTRE9 is specific to Flavnonexudans species and has likely been

353 recruited to the centromere only after the divergence of $V$. nubilum (Fig. 5a; Fig. S6-7).

354 Since VdLTRE9 occurs only in few Verticillium species, we assessed to which extent

355 other repetitive elements contribute to centromere organization. We analyzed the repeats

356 identified by de novo repeat predictions for each of the Verticillium species. Centromeres in all

357 species are AT- and repeat-rich (Fig. S6a-b), and some repeats occur in high frequency or nearly

358 exclusively at centromeres in species that lack VdLTRE9 (Table S1e). However, in contrast to

359 VdLTRE9, these repeats cover only a minority (typically less than 10\%) of the centromeres

360 (Table S1e). Sequence similarity-based cluster analyses of the de novo repeat consensus

361 sequences revealed that divergent repeat families contribute to Verticillium centromere

362 organization (Fig. S8). Thus, in contrast to VdLTRE9 in most Flavnonexudans species, we could

363 not identify any additional repeat family as a hallmark of centromeres in other Verticillium

364 species. 

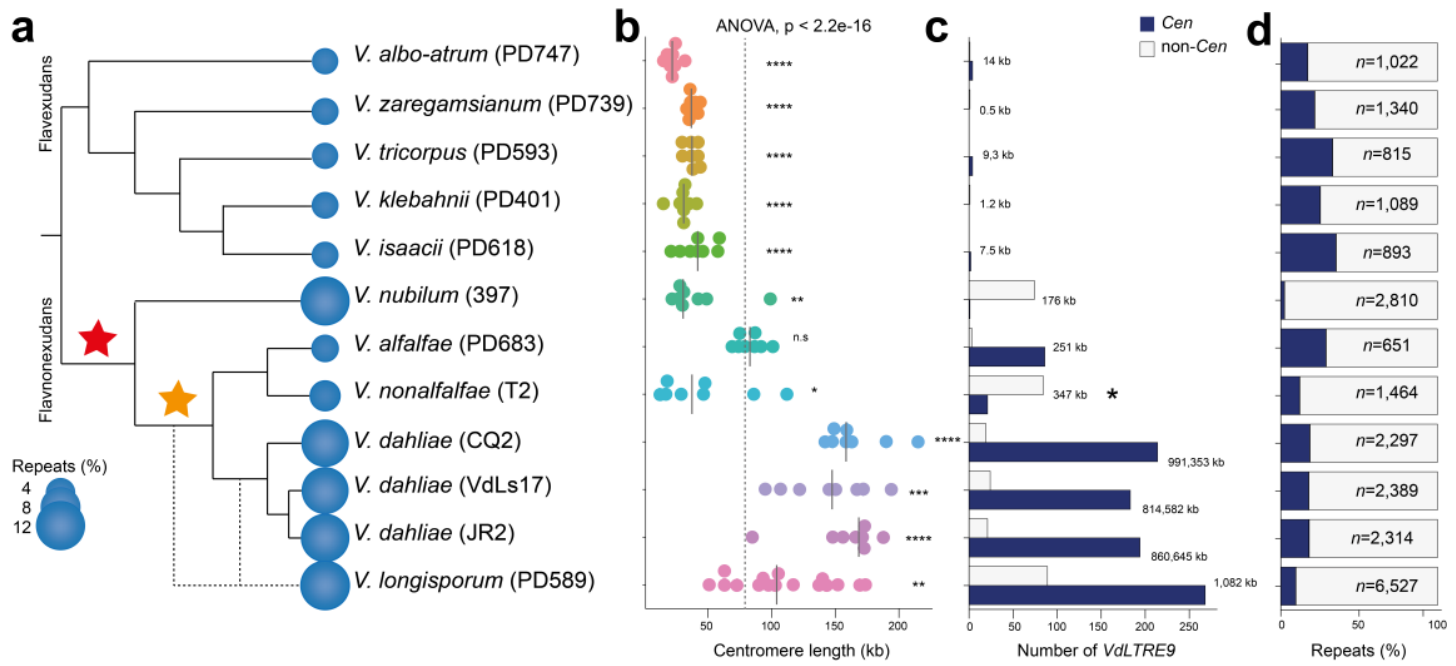

e
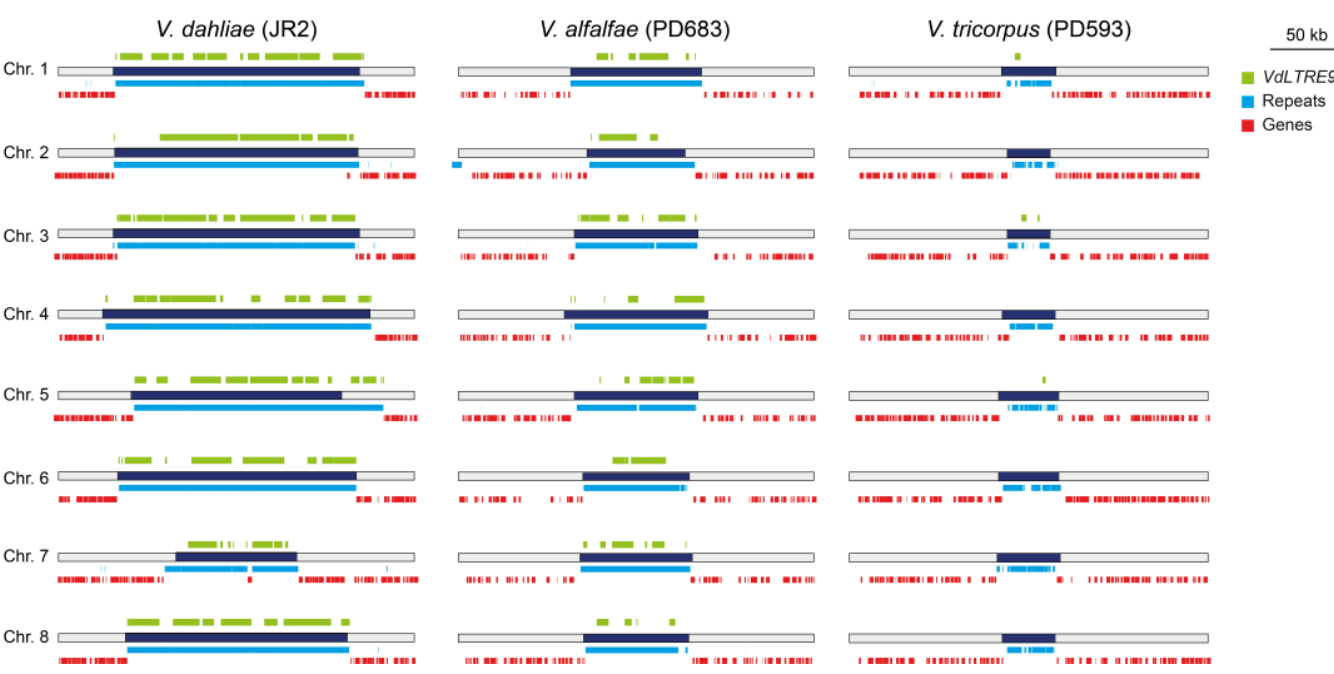

- Repeas
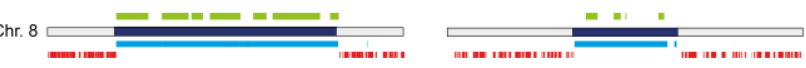

Figure 5 - Evolution of centromeres in the genus Verticillium. (a) Relationship of the ten members of the genus Verticillium. The predicted repeat content for each of the genomes is indicated (see Table S3 for details). The red star indicates the acquisition of VdLTRE9 in the Flavnonexudans clade while the yellow star indicates the recruitment of VdLTRE9 into centromeres. (b) Comparison of estimated centromere lengths (in $\mathrm{kb}$ ) in the different Verticillium spp. Each dot represents a single centromere and the line represents the median size. (c) The number of (partial) VdLTRE9 matches identified in centromeres (Cen; dark blue) and across the genome (non-Cen; light grey). The asterisk indicates the high number of VdLTRE9 elements in unassigned contigs for Verticillium nonalfalfae strain T2 (see text for details). (d) Proportion of predicted repeat content localized at centromeres (Cen; dark blue) and across the genome (nonCen; light grey). (e) Schematic overview of the eight centromeric regions (250 kb) in Verticillium dahliae strain JR2, and Verticillium alfalfae strain PD683 and Verticillium tricorpus stain PD593 as representatives for clade Flavnonexudans and clade Flavexudans, respectively. The centromeres are indicated by dark blue bars. The predicted genes (red) and repeats (light blue) are shown below each centromere, and location of (partial) VdLTRE9 matches (light green) are shown above each centromere. Global statistical differences for the centromere sizes was calculated using one-way ANOVA, and differences for each species compared to the overall 
mean were computed using unpaired T-tests; p-values $<0.0001$ : ****, p-values $<0.001$ : ***, pvalues < 0.01 : **, p-values < $0.05: *$.

\section{Centromeres contribute to Verticillium karyotype evolution}

387 We previously used fragmented genome assemblies to identify chromosomal rearrangements

388 during Verticillium evolution (8, 35, 40). We hypothesize that centromeres might have

389 contributed to these chromosomal rearrangements. To identify genome rearrangements and to

390 trace centromeres during Verticillium evolution, we used the pseudo-chromosomes of the haploid

391 Verticillium species to reconstruct ancestral chromosomal configurations using AnChro (Fig 6a)

392 (56). We reconstructed all potential ancestors that predominantly had eight chromosomes and

$393 \sim 8,000$ genes (Figs. S9a-b), yet the number of ancestral chromosomes and genes varied when

394 approaching the last common ancestor (Figs. S9a-b). By balancing the number of reconstructed

395 chromosomes and genes, we identified a single most parsimonious ancestral genome with eight

396 chromosomes and $~ 8,500$ genes (Fig. 6a; Fig. S9c), except for the last common ancestor within

397 the clade Flavexudans clade that had eight major chromosomes and two additional

398 'chromosomes' with only six and two genes (Fig. S9d). As these two smaller 'chromosomes'

399 likely do not represent genuine chromosomes, we conclude that all of the ancestral genomes,

400 similar to the extant haploid Verticillium genomes, had eight chromosomes (Fig. 6a). Confirming

401 our previous report (40), we observed in total 198 chromosomal rearrangements (124 inversions

402 and 74 translocations) (Fig. 6a). The number of chromosomal rearrangements is lower than

403 previously recorded and we did not observe any chromosomal fusion or fission events, which is

404 likely the result of the drastically improved genome assemblies, but the rearrangement signal on

405 each branch is sufficient to nevertheless recapitulate the known Verticillium species phylogeny

406 (Fig. S9e). Importantly, we observed 17 genomic rearrangements that occurred at, or in close

407 proximity (within $\sim 15$ genes up or downstream) to, centromeres, both in extant Verticillium

408 species as well as in the ancestors (Fig. 6). For example, at the branch from the last common

409 ancestor (VA; Fig. 6a) to the ancestor of the clade Flavexudans (B1; Fig. 6a), two centromere- 
410 associated translocations (between the ancestral chromosome 2 and 6) led to the formation of two

411 rearranged chromosomes. In total, we observed that five out of the eight ancestral centromeres

412 were associated with a chromosomal rearrangement at one point during evolution (Fig. 6a).

413 Nevertheless, comparisons of protein-coding genes that flank centromeres show that these are

414 syntenic in most extant species. Similarly, none of the recent chromosomal rearrangements

415 observed between $V$. dahliae strains is associated with centromeres (Figs. 4a-b, 6a). Thus, while

416 chromosomal rearrangements involving centromeres occurred during evolution, they do not

417 account for the majority of the karyotype variation between extant Verticillium species. 
bioRxiv preprint doi: https://doi.org/10.1101/2020.06.29.179234; this version posted June 30, 2020. The copyright holder for this preprint (which was not certified by peer review) is the author/funder, who has granted bioRxiv a license to display the preprint in perpetuity. It is made available under aCC-BY-ND 4.0 International license.

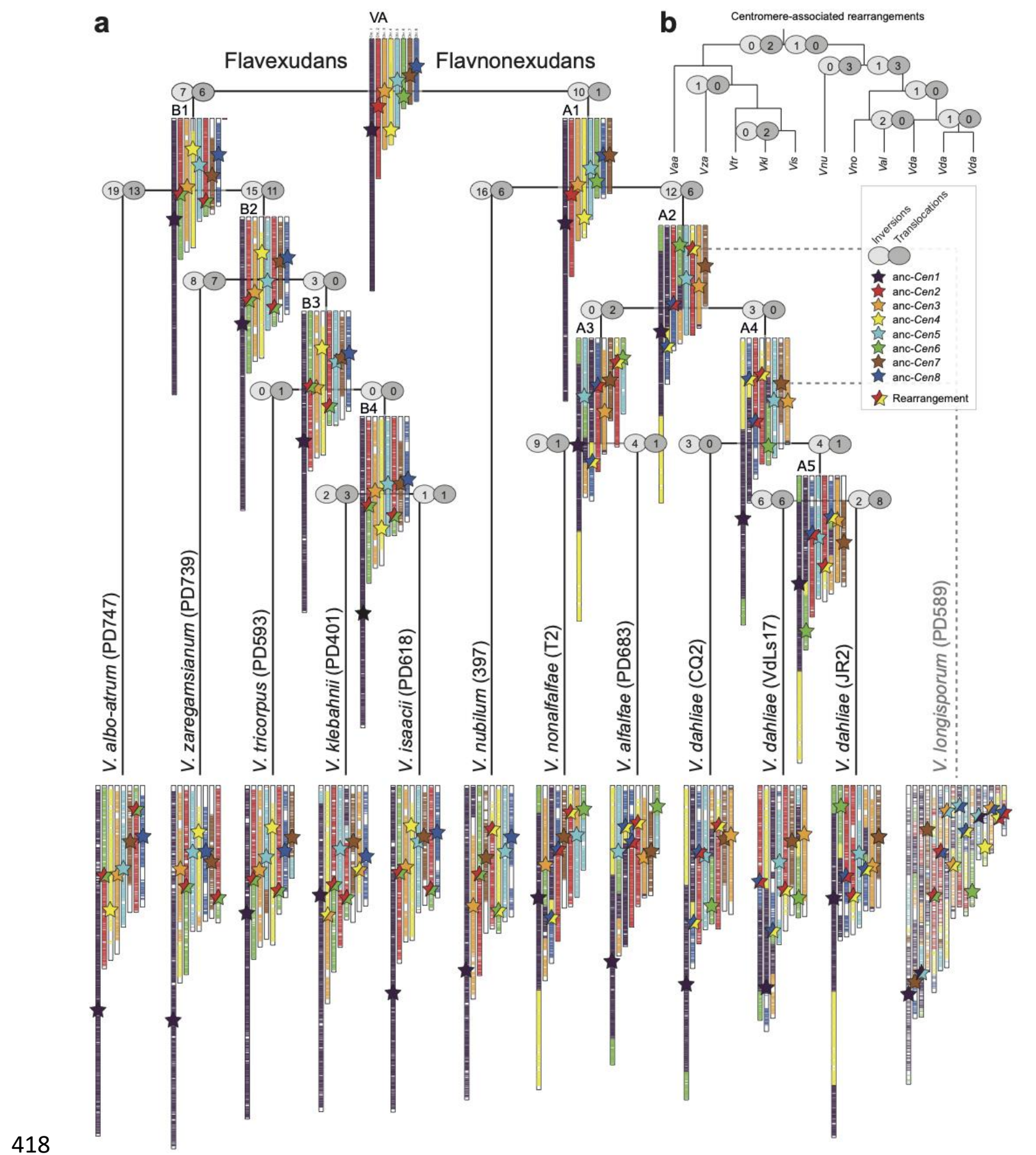

Figure 6 - Centromeres contribute to karyotype evolution in Verticillium. (a) Relationship of the ten members of the genus Verticillium. The allodiploidization event forming $V$. longisporum is indicated by dashed lines $(38,57)$. The chromosomal evolution within the haploid members of the genus was reconstructed using AnChro (56). The chromosomal structure of the nine species is shown in relation to the last common ancestor of the genus. The approximate locations of the centromeres are indicated by stars. The number of chromosomal rearrangements (inversions and translocations) are displayed for each branch, and centromeres that co-localize in proximity to 426 chromosomal rearrangements are highlighted by two-colored stars. (b) The number of major 
427 chromosomal rearrangements that occurred at, or in close proximity of, centromeres are shown

\section{DISCUSSION}

431 Centromeric regions are among the most rapidly evolving genomic regions (13-16, 29), yet

432 centromere evolution has only been systematically studied in few fungi $(11,12,16,29)$. Here, we

433 took advantage of the fungal genus Verticillium and used a combination of genetic and genomic

434 strategies to identify and characterize centromere organization and evolution. Verticillium

435 centromeres are characterized as large regional centromeres that are repeat-rich and embedded in

436 heterochromatin. We furthermore show that centromeres contribute to the karyotype evolution of

437 Verticillium. Finally, we demonstrate that VdLTRE9 is a hallmark of centromeres in some

438 Verticillium species, while species that lack VdLTRE9 display a divergent repeat content.

439 Centromeres in fungi, plants, and animals co-localize within the nucleus $(15,51-55,58)$,

440 a phenomenon that can be exploited for their identification $(51,52)$. Here, we used Hi-C to first

441 establish chromosome-level genome assemblies and subsequently identify centromeres in every

442 Verticillium species, and we demonstrate that centromere locations are in agreement with CenH3-

443 binding. While we obtained chromosome-level genome assemblies for all species, Hi-C

444 scaffolded genome assemblies could still contain partially collapsed repeats and assembly gaps, in

445 particular for short-read assemblies (59). With the exception of $V$. nonalfalfae, we observed only

446 few sequencing gaps and no evidence that would point to collapsed repeats at centromeres,

447 suggesting that the inferred centromeres are of high quality. Verticillium centromere sizes differ,

448 which is likely not driven by assembly artefacts, and centromeres in most Verticillium species are

449 larger than in Z. tritici (27), C. neoformans, M. oryzae, or Fusarium graminearum $(13,16,29)$,

450 yet smaller than in $N$. crassa (25). Species of the Flavexudans clade typically encode fewer

451 repeats than species of the clade Flavnonexudans clade $(32,40,60)$, and $V$. nubilum, $V$.

452 longisporum, and $V$. dahliae are particularly rich in repeats when compared with other 
453 Verticillium species (32, 39-41, 60). Thus, increased centromere sizes positively correlate with

454 overall increased repeat contents.

455 Using fragmented genome assemblies, we previously identified chromosomal

456 rearrangements during Verticillium evolution $(8,35,40)$ that were thought to have contributed to

457 genetic diversity and adaptation in the absence of sexual recombination $(7,35,40)$. Chromosome-

458 level genome assemblies for an entire genus enabled unprecedented analyses of the karyotype

459 evolution over longer evolutionary timescales. Here, we observed extensive chromosomal

460 rearrangements and provide evidence that some rearrangements at centromeres contributed to

461 karyotype evolution, most of which occurred early during the divergence of Verticillium.

462 Chromosomal rearrangements at centromeres occur in the fungal yeasts Candida, Cryptococcus,

463 and Malassezia $(11,12,61)$, and synteny breakpoints have been identified between mammals and

464 chicken (62), suggesting that centromeres often contribute to karyotype evolution. The emergence

465 of chromosomal rearrangements at centromeres could be facilitated by their repeat-rich nature

466 (11, 12). For example, centromeres in Malassezia are enriched with an AT-rich motif that could

467 facilitate replication fork stalling, which leads to double strand DNA breaks (11). Repeats

468 localized outside of centromeres in V. dahliae contribute to chromosomal rearrangements (8), and

469 thus it seems plausible that centromeric repeats similarly contribute to chromosomal

470 rearrangements. Chromosomal rearrangements often do not only lead to changes in chromosome

471 organization but also in chromosome number $(11,12)$. While we observed chromosomal

472 rearrangements, all extant and ancestral genomes contained eight chromosomes, suggesting that

473 eight chromosomes are a stable configuration for all Verticillium species.

474 Centromere position and function are thought to be driven by the protein complement

475 (e.g. CenH3 localization) and by heterochromatin formation rather than by specific DNA

476 sequences $(13,15,63)$. In $V$. dahliae, we observed the co-occurrence of CenH3 with H3K9me3

477 and DNA methylation. This suggests that DNA methylation, as previously reported in N. crassa 
478 and in $C$. neoformans $(16,25)$, is also a feature of centromeric DNA in $V$. dahliae. Co-

479 localization of CenH3 with $\mathrm{H} 3 \mathrm{~K} 9 \mathrm{me} 2 / 3$ and DNA methylation has been reported for $N$. crassa

480 (25) and C. neoformans (16). In contrast, H3K9me3 and H3K27me3 are absent from centromeres

481 in Z. tritici (27). H3K4me2 borders most centromeres in Z. tritici (27), and is associated with

482 centromeres in $S$. pombe and some animals and plants (64-67). H3K4me2 has not been observed

483 at centromeres in most fungi, including $V$. dahliae, and in the oomycete $P$. sojae (30). Changes in

484 heterochromatin in $N$. crassa leads to altered CenH3 positioning (25), suggesting that

485 heterochromatin is similarly required for centromere maintenance and function in V. dahliae.

486 Elevated AT-levels in repeat-rich heterochromatic regions can be caused by RIP mutations (15,

$48725,26,42)$. RIP-like mutations have been previously reported in some repeats in V. dahliae (36,

488 44), and we observed strong RIP signals at centromeres. Due to its presumably asexual nature (7),

489 the occurrence of RIP in $V$. dahliae is controversial $(8,43,44)$. Noteworthy, mutational signatures

490 resembling RIP have recently been observed in Z. tritici propagated through mitotic cell

491 divisions, pointing to the existence of a mitotic version of a RIP-like process (42). Thus, we

492 conclude that RIP was an active process in $V$. dahliae at some point in evolution, or that RIP-like

493 processes outside of the sexual cycle occur in $V$. dahliae.

494 Centromeres are often enriched for a variety of different retrotransposons and other

495 repetitive elements $(15,16,25,29,30)$. We similarly observed that centromeres in all Verticillium

496 species are repeat-rich. Repeats and their remnants identified at centromeres typically also occur

497 outside of centromeres, as observed in M. oryzae (29) and N. crassa (25). Strikingly, we observed

498 that a single repetitive element, VdLTRE9, is strongly associated with centromeres in some

499 Verticillium species, which to our knowledge, has only been observed in the fungus Cryptococcus

500 where centromeres contain six retrotransposons (Tcn1-6) that nearly exclusively occur at

501 centromeres (16). Similarly, centromeres of the oomycete plant pathogen Phytophthora sojae

502 contain multiple types of repeats, but they are enriched for a single element called CoLT (Copia- 
503 Like Transposon) (30). The strong associations of specific repeats to centromeres could directly

504 or indirectly link these elements to centromere function. Functional centromeres as observed here

505 are also heterochromatic and contain CenH3. AT-rich repetitive elements can direct

506 heterochromatin formation via DNA methylation and H3K9me3 deposition in N. crassa $(45,68)$,

507 a phenomenon that can also occurs at repeats outside of centromeres (45). Heterochromatin

508 occurs at centromeres but also at repeat-rich regions outside of centromeres in V. dahliae, thus the

509 repeat-rich nature of centromeres is likely not sufficient to direct CenH3 deposition. In S. pombe

510 heterochromatin formation is directed by short interfering RNAs (siRNA) derived from flanking

511 repetitive elements via $\operatorname{RNAi}(69,70)$, and RNAi and heterochromatin mediate CenH3

512 localization at centromeres $(71,72)$. RNAi is also important for centromere maintenance and

513 evolution in Cryptococcus, as RNAi deficient species have smaller centromeres than RNAi

514 proficient ones (16). Interestingly, centromere-specific elements (Tcnl-6) in RNAi proficient

515 species are typically full-length elements while only remnants can be found in RNAi deficient

516 species, which could be caused by recombination between elements (16). In Verticillium,

517 centromere size differences correlate with increase of repeat content and the recruitment of

518 VdLTRE9, which is highly fragmented and likely non-active. Furthermore, even though key

519 components of the RNAi machinery exist in at least some Verticillium species (73), we know

520 very little about its biological functions. Similarly, to $C$. neoformans, we observed no

521 transcriptional activity of VdLTRE9 or any other repeat at centromeres, but it is unclear if this

522 silencing is mediated by RNAi, is a consequence of their heterochromatic nature, is due to their

523 fragmentation, or a combination of these. Ultimately, unravelling how specific elements

524 contribute to centromere identify necessitates future experiments. VdLTRE9 occurs only in some

525 Verticillium species and has likely been recruited to centromeres subsequent to the divergence of

526 V. nubilum. Conversely, these observations raise further questions on the roles of repeats and

527 mechanisms of centromeric identity in species without VdLTRE9. Repeats are important drivers

528 of Verticillium genome evolution and function $(8,36)$, and here we highlight their contributions 
529 to centromere diversity within the fungal genus Verticillium. Our analyses provide the framework

530 for future research into the diversity or convergence of mechanisms establishing centromere

531 identity and functioning in fungi. 


\section{MATERIAL \& METHODS}

\section{Construction of Verticillium dahliae transformants expressing FLAG-tagged CenH3}

$534 \mathrm{CenH} 3$ and $\mathrm{H} 3$ homologs were identified in the predicted proteomes of $V$. dahliae strain JR2 (32)

535 and selected other fungi through a BLAST sequence similarity search (blastp v2.9.0+; default

536 settings, e-value cutoff 1e-20) (74, 75) using the $N$. crassa CenH3 (Q7RXR3) and H3 (P07041)

537 sequences as queries. Missing homologs of $\mathrm{CenH} 3$ or $\mathrm{H} 3$ were identified using manual BLAST

538 (tblastn v2.9.0+; default settings) $(74,75)$ and exonerate (v2.2.0; default settings) (76) searches

539 against the genome sequences. Protein sequences of selected $\mathrm{CenH} 3$ and $\mathrm{H} 3$ proteins were

540 aligned using mafft (v7.271; default settings, LINSi) (77). A phylogenetic tree was inferred with

541 maximum-likelihood methods implemented in IQ-tree (v1.6.11) (78) and robustness was assessed

542 by 1,000 rapid bootstrap replicates.

543 To construct the N-terminally FLAG-tagged CenH3 strain of $V$. dahliae, a recombinant

544 DNA fragment was constructed into the binary vector PRF-HU2 (79) or PRF-GU2 for

545 homologous recombination. The CenH3 locus, from $V$. dahliae strain JR2, was amplified as 3

546 fragments with overlapping sequences (Table S1f). The 5' most fragment containing the

547 promoter was amplified using primers $\mathrm{A}+\mathrm{B}$, the $\mathrm{ORF}$ with primers $\mathrm{C}+\mathrm{D}$, the Hyg promoter and

548 ORF with primers $\mathrm{E}+\mathrm{F}$, and the 3' end of the CenH3 locus with primers $\mathrm{G}+\mathrm{H}$. The four fragments

549 were combined by overlap PCR using primers $\mathrm{A}+\mathrm{H}$ and cloned into a PspOMI and SphI

550 linearized vector using Gibson Assembly. The vector construction was confirmed by Sanger

551 sequencing. Vectors were transformed to Verticillium with Agrobacterium-mediated

552 transformation (80). Correct homologous recombination and replacement at the CenH3 locus was

553 verified by PCR amplification using primer I+J (Fig. S1b, Table S1f). Correct translation of the

554 recombinant protein was assessed using Western analyses with anti-FLAG antibody (Fig. S1c).

555 Briefly, proteins were extracted from 5-day old cultures grown in $100 \mathrm{ml}$ Potato Dextrose Broth

556 at $22^{\circ} \mathrm{C}$ with continuous shaking at $120 \mathrm{rpm}$. Mycelium was collected by straining over a double 
557 layer of miracloth and subsequently snap-frozen in liquid nitrogen and ground with a mortar and

558 pestle using liquid nitrogen. Approximately $0.3 \mathrm{~g}$ of ground mycelium was resuspended in 600

$559 \mu \mathrm{L}$ protein extraction buffer (50 mM HEPES pH 7.5, $150 \mathrm{mM} \mathrm{NaCl}, 1 \mathrm{mM}$ EDTA, $1 \%$ glycerol,

$560 \quad 0.02 \%$ NP-40, 2 mM Phenylmethanesulfonyl fluoride (PMSF), $100 \mu \mathrm{M}$ Leupeptin, $1 \mu \mathrm{g} / \mathrm{mL}$

561 Pepstatin), briefly vortexed, incubated on ice for $15 \mathrm{~min}$ and centrifuged at $4^{\circ} \mathrm{C}$ at $8,000 \mathrm{~g}$ for 3

562 min. The supernatant was collected by transferring $20 \mu \mathrm{L}$ to a new tube to serve as the input

563 control and the remaining $\sim 500 \mu \mathrm{L}$ was transferred to a fresh microcentrifuge tube with $15 \mu \mathrm{L}$ of

564 Anti-FLAG M2 affinity gel (catalog number A2220, Sigma-Aldrich, St. Louis, Missouri, United

565 States) and incubated while rotating at $4^{\circ} \mathrm{C}$ for $1 \mathrm{~h}$. Samples were centrifuged at $5,000 \mathrm{~g}, 4^{\circ} \mathrm{C}$ for

$5663 \mathrm{~min}$, after which the supernatant was discarded. Samples were washed with $500 \mu \mathrm{L}$ of lysis

567 buffer, and the centrifugation and washing were repeated three times. Protein was eluted from the

568 resin by adding $15 \mu \mathrm{L}$ of lysis buffer, $20 \mu \mathrm{L}$ of $2 \mathrm{x}$ Laemmli loading buffer (4\% SDS, $20 \%$

569 glycerol, $0.004 \%$ bromophenol blue, $125 \mathrm{mM}$ Tris HCL pH 6.8) and boiled at $95^{\circ} \mathrm{C}$ for 3 min.

570 Protein samples were separated on a $12 \%$ polyacrylamide gel, and subsequently transferred to

571 PVDF membranes, blocked in 5\% BSA, washed twice in TBST, and incubated with 1:3500 anti-

572 FLAG antibody (monoclonal anti-FLAG M2; Merck KGaA, Darmstadt, Germany).

573

574 Chromatin immunoprecipitation followed by high-throughput sequencing (ChIP-seq)

575 For each $V$. dahliae genotype, one million spores were added to $100 \mathrm{ml}$ Potato Dextrose Broth

576 and incubated for 7 days at $22^{\circ} \mathrm{C}$ with continuous shaking at $120 \mathrm{rpm}$. Mycelium was collected by

577 straining over a double layer of miracloth and subsequently snap-frozen in liquid nitrogen and

578 ground with a mortar and pestle using liquid nitrogen. All ground material (0.5-1 gram per

579 sample) was resuspended in $4 \mathrm{~mL}$ ChIP Lysis buffer (50 mM HEPES-KOH pH7.5, $140 \mathrm{mM}$

$580 \mathrm{NaCl}, 1 \mathrm{mM}$ EDTA, $1 \%$ Triton X-100, 0.1\% NaDOC) and dounced 40 times in a $10 \mathrm{~cm}^{3}$ glass

581 tube with tightly fitting pestle on 800 power with a RZR50 homogenizer (Heidolph, Schwabach,

582 Germany), followed by five rounds of 20 seconds sonication on ice with 40 seconds of resting in 
583 between rounds with a Soniprep 150 (MSE, London, UK). Samples were redistributed to $2 \mathrm{~mL}$

584 tubes and pelleted for $2 \mathrm{~min}$ at maximum speed in a tabletop centrifuge. Supernatants were

585 pooled per sample in a $15 \mathrm{~mL}$ tube together with $25 \mu \mathrm{L} \alpha$-FLAG M2 magnetic beads (Sigma-

586 Aldrich, St. Louis, Missouri, United States), incubated overnight at $4{ }^{\circ} \mathrm{C}$ and continuous rotation.

587 Beads were captured on a magnetic stand and washed with wash buffer $(50 \mathrm{mM}$ Tris $\mathrm{HCl} \mathrm{pH} 8,1$

588 mM EDTA, $1 \%$ Triton X-100, $100 \mathrm{mM} \mathrm{NaCL}$ ), high-salt wash buffer (50 mM Tris $\mathrm{HCl} \mathrm{pH} 8,1$

589 mM EDTA, 1\% Triton X-100, $350 \mathrm{mM} \mathrm{NaCL})$, LiCl wash buffer (10 mM Tris HCl pH8, $1 \mathrm{mM}$

590 EDTA, $0.5 \%$ Triton X-100, $250 \mathrm{mM} \mathrm{LiCl})$, and TE buffer (10 mM Tris $\mathrm{HCl} \mathrm{pH} 8,1 \mathrm{mM}$ EDTA).

591 Chromatin was eluted twice from beads by addition of $100 \mu \mathrm{L}$ pre-heated TES buffer (100 mM

592 Tris $\mathrm{HCl} \mathrm{pH} 8,1 \%$ SDS, $10 \mathrm{mM}$ EDTA, $50 \mathrm{mM} \mathrm{NaCl}$ ) and 10 minutes incubation at $65^{\circ} \mathrm{C} .10$

$593 \mathrm{mg} / \mathrm{mL} 2 \mu \mathrm{L}$ Proteinase $\mathrm{K}$ was added and incubated at $65^{\circ} \mathrm{C}$ for 5 hours, followed chloroform

594 extraction. DNA was precipitated by addition of 2 volumes $100 \%$ ethanol, $1 / 10^{\text {th }}$ volume $3 \mathrm{M}$

$595 \mathrm{NaOAc}$ pH 5.2 and $1 / 200^{\text {th }}$ volume $20 \mathrm{mg} / \mathrm{mL}$ glycogen, and overnight incubation at $-20^{\circ} \mathrm{C}$.

596 Sequencing libraries were prepared using the TruSeq ChIP Library Preparation Kit

597 (Illumina, city, country) according to the manufacterer's instructions, but without gel purification

598 and with use of the Velocity DNA Polymerase (BioLine, Luckenwalde, Germany) for 12 cycles

599 of amplification for the FLAG-CenH3. H3K4me2 ChIP was performed as described previously

600 (36), using an $\alpha-H 3 K 4 m e 2$ antibody (\#39913, ActiveMotif; Carlsbad, California, United States).

601 Single-end (125 bp) sequencing was performed on the Illumina HiSeq2500 platform at KeyGene

602 N.V. (Wageningen, the Netherlands).

603

604 Chromatin confirmation capturing followed by high-throughput sequencing (Hi-C)

605 We determined the inter- and intra-chromosomal contact frequencies using Hi-C in $V$. dahliae

606 strains CQ2, JR2, and VdLs17, as well as in V. albo-atrum strain PD747, V. alfalfae strain

607 PD683, V. isaacii strain PD618, V. klebahnii strain PD401, V. longisporum strain PD589, V. 
nonalfalfae strain T2, V. nubilum strain 397, V. tricorpus strain PD593, and V. zaregamsianum

609 strain PD739. For each strain, one million spores were added to $400 \mathrm{~mL}$ Potato Dextrose Broth

610 and incubated for 6 days at $22^{\circ} \mathrm{C}$ with continuous shaking at $120 \mathrm{rpm}$. Mycelium was collected by

611 straining over double layer miracloth and $300 \mathrm{mg}$ (fresh weight) was used as input for generating

612 Hi-C sequencing libraries with the Proximo Hi-C kit (Microbe) (Phase Genomics, Seattle, WA,

613 USA), according to manufacturer's instructions. Hi-C sequencing libraries of V. dahliae strains

614 CQ2, JR2. and VdLs17 were paired end (2x125 bp) sequenced on the Illumina HiSeq2500

615 platform at KeyGene N.V. (Wageningen, the Netherlands). Hi-C sequencing libraries of the other

616 Verticillium species were paired-end (2x150 bp) sequenced on the NextSeq500 platform at USEQ

617 (Utrecht, the Netherlands).

619 In vitro transcriptome profiling using RNA-seq

620 RNA sequencing of $V$. albo-atrum strain PD747, V. isaacii strain PD618, V. klebahnii strain

621 PD401, V. longisporum strain PD589, V. nonalfalfae strain T2, V. nubilum strain 397, $V$.

622 tricorpus strain PD593, and V. zaregamsianum strain PD739 as described previously (36). Single-

623 end (50 bp) sequencing was performed on the BGISeq500 platform at BGI (BGI Hong Kong).

\section{Analyses of high-throughput sequencing data}

626 High-throughput sequencing libraries (Table S1a) have been analyzed as follows: Illumina reads

627 were quality-filtered and trimmed using trimmomatic (version 0.36) (81). Sequencing reads were

628 trimmed and filtered by removing Illumina TruSeq sequencing adapters (settings seed

629 mismatches 2, palindrome clip threshold 30, and simple clip threshold 10), removal of low-

630 quality leading or trailing bases below quality 5 and 10, respectively, and 4-base sliding window

631 trimming and cutting when average quality per base dropped below 15. Additionally, filtered and

632 trimmed reads $<90$ nt were removed from further analyses. Filtered and trimmed reads were

633 mapped to the corresponding genome assembly with Bowtie2 (default settings) (82), and mapping 
634 files were converted to bam-format using samtools (v 1.8) (83). Genomic coverage was

635 determined using deepTools (v3.4.1; bamCoverage) (84) by extending sequencing reads to 147

636 bp followed by RPGC normalization with a bin-size of 1,000 bp and smoothening of 3,000 bp. To

637 assess between sample variability, we used deepTools (v3.4.1, plotPCA) (84) to generate

638 principle component analyses. Furthermore, we employed deepTools (v3.4.1,

639 multiBigwigSummary) (84) to summarize genomic coverages of over genes, repetitive elements,

640 and genomic windows (5 kb windows with 500 bp slide). Genomic regions enriched for FLAG-

641 CenH3 were identified using MACS2 (v2.1.1) (broad peak option; broad cutoff 0.0025) (85).

642 To determine DNA (cytosine) methylation, we utilized sequencing data of bisulfite treated

643 genomic DNA previously generated for $V$. dahliae strain JR2 (36). Sequencing reads were

644 mapped to the $V$. dahliae strain JR2 genome assembly as previously described (36).

645 Subsequently, the number of reads supporting cytosine methylation in CG-context were extracted,

646 and weighted CG-methylation levels were calculated over genes, repetitive elements, and

647 genomic windows (5 kb window size with $500 \mathrm{bp}$ slide) (86); weighted CG-methylation was

648 defined as the sum of reads supporting cytosine methylations divided by the sum of all reads

649 occurring at all CG sites in the respective regions. Sites with less than four reads were not

650 considered.

651 To improve the genome assemblies of the Verticillium species, we mapped Hi-C 652 sequencing reads to genome assemblies of $V$. dahliae strain CQ2, $V$. albo-atrum strain PD747, $V$.

653 alfalfae strain PD683, V. isaacii strain PD618, V. klebahnii strain PD401, V. longisporum strain

654 PD589, V. nonalfalfae strain T2, V. nubilum strain 397, V. tricorpus strain PD593, and V.

655 zaregamsianum strain PD739 using Juicer (v1.6) with early stage setting (87). The contact

656 matrices generated by juicer were used by the 3D de novo assembly (3D-DNA) pipeline (88)

657 (v180922) with a contig size threshold of 1000bp to eliminate mis-joints in the previous

658 assemblies and to generate improved assemblies. The genome assemblies were manually 
659 improved using Juicebox Assembly Tools (JBAT) (v1.11.08) (89) and improved genome

660 assemblies were generated using the 3D-DNA post-review asm pipeline (88). Centromere

661 locations were determined using a 1 kb-resolution contact matrix in JBAT, by identifying a region

662 per chromosome that displays strong inter-chromosomal interactions, yet weak intra-

663 chromosomal interactions (see Figure S12, S13).

664 To assess potential repeat collapses during genome assemblies at centromeric regions, we 665 mapped previously generated short-read data V. dahliae strain JR2 and VdLs17, V. albo-atrum 666 strain PD747, V. alfalfae strain PD683, V. isaacii strain PD618, V. klebahnii strain PD401, V. 667 longisporum strain PD589, Verticillium nonalfalfae strain T2, V. tricorpus strain PD593, and V. 668 zaregamsianum strain PD739 $(39,40,90,91)$ to the genome assemblies using BWA (v0.7.17; 669 mem) (83). We first used bedtools (v2.29.2) (92) to identify few genomic regions with $>500 \mathrm{x}$ 670 coverage. We then applied deepTools (v3.4.1, computeGCBias) (84) to compute GC biases of 671 read depth across the genome, excluding the identified high coverage regions, and used 672 deepTools (v3.4.1, correctGCBias) (84) to correct GC biases, which addresses known biases in 673 sequencing library preparation to ensure even read coverage throughout the genome irrespective 674 of their base composition (93). We used deepTools (v3.4.1, bamCoverage, bins $50 \mathrm{bp}$, CPM 675 normalization) (84) to obtain the read coverage throughout the genome, excluding regions 676 containing sequence assembly gaps (Ns). Assuming that collapsed repeats would lead to a local 677 increase in read depth, we used the ratio of the average read coverage at the centromeres and 678 outside of the centromere at each chromosome to correct the inferred centromere sizes. To further 679 validate the genome assembly of regions identified as centromeres of $V$. dahliae strain JR2, the 680 genome assembly was compared to the previously generated optical map (35) using MapSolver (v 681 3.2; OpGen, Gaithersburg, MD).

682 The transcriptional activity for genes and repetitive elements in $V$. dahliae strain JR2 was 683 assessed in vitro (in Potato Dextrose Broth) using previously generated deep transcriptome 
684 datasets (36). To this end, single-end sequencing reads of three biological replicates were mapped

685 to the $V$. dahliae strain JR2 genome assembly (32) using STAR (v2.4.2a; max. intron size $1 \mathrm{~kb}$

686 and outFilterMismatchNmax to 5) (94). The resulting mapped reads were summarized per

687 genomic feature (gene or repeat) using summarizeOverlaps (95), converted to counts per million

$688(\mathrm{cpm})$ mapped reads, and averaged over the three biological replicates.

690 Sequence analyses of Verticillium genome assemblies, centromeres, repeat and gene content

691 Repetitive elements in the genomes of $V$. dahliae strains JR2, VdLs17 and CQ2 (32, 33) were

692 identified as previously described (36). Briefly, repetitive elements were identified in each

693 genome independently using a combination of LTRharvest (96) and LTRdigest (97) followed by

694 identification of RepeatModeler. Identified repeats in the different $V$. dahliae strains were

695 clustered into a non-redundant library that contained consensus sequences for each repeat family.

696 The repeat library was manually curated and annotated using PASTEC (98) or by sequence

697 similarity to previously identified and characterized repeat families $(32,44)$. Genome-wide

698 occurrences of repeat families were determined using RepeatMasker ( $\mathrm{v} 4.0 .9$; sensitive option and

699 cutoff 250), and the output was postprocessed using 'One code to find then all' (99). We only

700 considered matches to the repeat consensus library, and thereby excluded simple repeats and low-

701 complexity regions.

De novo gene and repeat annotation for the Hi-C-improved Verticillium genome

703 assemblies, and for V. dahliae strains JR2 and VdLs17 as a comparison was performed using the

704 funannotate pipeline (100). Briefly, repetitive elements were first de novo identified using

705 RepeatModeler and masked for gene prediction using RepeatMasker. Subsequently, gene

706 prediction parameters were estimated using in vitro RNA-seq data (see above for details;

707 exception: $V$. alfalfae for which no RNA-seq data was available, $V$. nonalfalfae for which

708 publicly available RNA-seq data was used (90), and V. dahliae strain JR2 for which in addition to 
709 the in vitro RNA-seq data generated in this study, also previously generated in vitro (xylem sap

710 and half-MS; (36)) as well as long-read nanopore cDNA data (101) was used). Based on the gene

711 prediction parameters, gene prediction was performed with funannotate using a combination of $a b$

712 initio gene predictors, consensus predictions were obtained using Evidencemodeler (v1.1.1)

713 (102), and gene predictions were adjusted using information from the RNA-seq data. Repeat

714 annotation for each genome assembly was based on the de novo repeat family consensus

715 sequences obtained with funannotate. Genome-wide occurrences of these repeat families as well

716 as previously defined repeat families for $V$. dahliae (see above) were determined using

717 RepeatMasker (v 4.0.9; sensitive option and cutoff 250), and the output was postprocessed using

718 'One code to find then all' (99). De novo repeat families overlapping with centromeres in the

719 different species were clustered using BLASTClust (v2.2.26; parameter '-S 60 -L 0.55 -b F -p F'),

720 and subsequently visualized using Cytoscape (v.3.8.0) (103). Next to RepeatMasker, genome-

721 wide occurrences of the previously determined VdLTRE9 $(32,36)$ were identified by BLAST

722 searches (blastn v2.9.0+; e-value cutoff 1e-5, no soft-masking and dust, fixed database size 10e6)

$723(74,75)$, and similarity between VdLTRE9 consensus sequences and the de novo predicted repeat

724 families was established using BLAST (blastn, e-value cutoff 1e-5, query coverage > 50\%, no

725 soft-masking and dust, fixed database size 10e6).

726 Repeat and gene density (V. dahliae strain JR2 and VdLs17 based on previous gene

727 annotation (101)), GC-content, and composite RIP index were calculated along the genome

728 sequence using sliding windows ( $5 \mathrm{~kb}$ window with $500 \mathrm{bp}$ slide). The composite RIP index

729 (CRI) was calculated according to Lewis et al. (45). CRI was determined by subtracting the RIP

730 substrate from the RIP product index, which are defined by dinucleotide frequencies as follows:

731 RIP product index $=\mathrm{TpA} / \mathrm{ApT}$ and the RIP substrate index $=(\mathrm{CpA}+\mathrm{TpG}) /(\mathrm{ApC}+\mathrm{GpT})$.

732 Overlaps between different genomic features (for example repetitive elements over centromeric 
733 regions) was assessed using bedtools (v2.29.2) (92). Genome-wide data was visualized using R

734 (104) with the packages ggplot2 (105), karyplotR (106), or Gviz (107), as well as EasyFig (108).

735 Whole-genome alignments between $V$. dahliae strains JR2, VdLs17, and CQ2 were

736 performed using NUCmer, which is part of the MUMmer package (v 3.1; --maxmatch) (109). To

737 remove short matches, we only considered alignments longer than $10 \mathrm{~kb}$. Ancestral genome

738 configurations were reconstructed using AnChro (56). We first determined the synteny

739 relationships between all possible pairs of haploid Verticillium genomes and two outgroup

740 genomes (Plectosphaerella cucumerina and Sodiomyces alkalinus) using SynChro with synteny

741 block stringency (delta parameter) ranging from 2-5 (110). We then obtained all ancestors by

742 calculating all possible pairs of genomes (G1 and $\mathrm{G} 2)$ and outgroups $\left(\mathrm{G} 3, . ., \mathrm{G}_{\mathrm{n}}\right)$ and by varying

743 the delta' (G1 and G2 comparisons) and delta", (G1/G3..G1/G $\mathrm{G}_{\mathrm{n}}$ and G2/G3..G2/G $\mathrm{G}_{\mathrm{n}}$ comparisons)

744 parameters for AnChro. We additionally reconstructed all ancestors starting from the extent

745 genomes in a sequential approach with multiple successive cycles of SynChro and AnChro (delta

746 parameters varied between 2-5). For each ancestor, we chose the optimal reconstructed by the

747 delta parameter combination (delta' and delta') that minimizes the number of reconstructed

748 chromosomes and rearrangements and at the same time maximizes the number of genes, both

749 guided by the most commonly observed number of chromosomes and genes in all

750 rearrangements. We obtained the number of large-scale rearrangements between reconstructed

751 ancestral genomes and the extent Verticillium genomes using ReChro with a delta parameter of 1

752 (56). The relationship between chromosomes of the reconstructed ancestors and the extent species

753 in relationship to the common ancestor is generated with SynChro with a delta parameter of 1

754 (110). A species phylogeny that uses synteny relationships computed by SynChro (see above) as

755 informative character between the Verticillium genomes and the outgroup genomes was

756 reconstructed using PhyChro (111). 


\section{Data availability}

759 ChIP-seq and Hi-C data were submitted to the Short Read Archive (SRA) under the accession

760 PRJNA641329 (Table S1a).

761

762 ACKNOWLEDGMENTS

763 Work in the laboratories of M.F.S and B.P.H.J.T. is supported by the Research Council Earth and

764 Life Sciences (ALW) of the Netherlands Organization of Scientific Research (NWO).

765 Furthermore, B.P.H.J.T. would like to acknowledge the Deutsche Forschungsgemeinschaft (DFG,

766 German Research Foundation) under Germany's Excellence Strategy - EXC 2048/1 - Project ID:

767 390686111. This work was supported in part by a European Molecular Biology Organization

768 postdoctoral fellowship (EMBO, ALTF 969-2013) and Human Frontier Science Program

769 Postdoctoral Fellowship (HFSP, LT000627/2014-L) to D.E.C. A portion of the work was also

770 carried out in the laboratory of D.E.C. under USDA-NIFA-PBI grant 2018-67013-28492. We

771 thank Utrecht Sequencing Facility for providing sequencing service and data. Utrecht Sequencing

772 Facility is subsidized by the University Medical Center Utrecht, Hubrecht Institute, Utrecht

773 University, and The Netherlands X-omics Initiative (NWO project 184.034.019). 


\section{SUPPLEMENTARY MATERIAL}

775 Figure S1 - (a) Phylogenetic analyses of the canonical H3 and the centromeric-specific CenH3 in

776 Verticillium dahliae (strain JR2) and other fungal genomes. (b-c) Transformation of the coding

777 sequence of N-terminally FLAG-tagged $\mathrm{CenH} 3$ directed by its native promoter at the CenH3

778 locus in Verticillium dahliae strain JR2. (b) Correct homologous recombination and replacement

779 at the CenH3 locus was verified by PCR amplification was assessed using PCR and (c) Correct

780 translation of the recombinant protein was assessed using Western Blot analyses with anti-FLAG

781 antibody. (d) Sequencing read coverage (RPGC normalization in $1 \mathrm{~kb}$ bins with $3 \mathrm{~kb}$

782 smoothening) from ChIP-seq experiments using FLAG-tag antibodies on two independent

783 transformants of Verticillium dahliae strain JR2 that express FLAG-tagged CenH3 and the wild-

784 type strain are mapped to the eight chromosomes of $V$. dahliae strain JR2 (32). Gene (red) and

785 repeat (blue) density are shown below each chromosome. (e) Principal component analysis of the

786 four FLAG-tag ChIP-seq samples (two wild-type and two CenH3-FLGA). (f) Comparison of the

787 centromeric regions with the identified centromeres highlighted as blue block in the genome

788 assembly of Verticillium dahliae strain JR2 with a previously generated optical map (35). Vertical

789 lines display corresponding (in silico) restriction sites and their alignment.

790 Figure S2 - Schematic overview of the eight chromosomes of Verticillium dahliae strain JR2

791 displaying different heterochromatin-associated chromatin modifications (mC, H3K9me3, and

792 H3K27me3) in relation to the centromeres. The different lanes display the CenH3-FLAG ChIP-

793 seq read coverage (RPGC normalization in $1 \mathrm{~kb}$ bins with $3 \mathrm{~kb}$ smoothening), the repeat-density,

794 the GC-content, the CRI as well as the weighted cytosine methylation (all summarized in $5 \mathrm{~kb}$

795 windows with 500 bp slide), and the normalized H3K9me3 and H3K27me3 ChIP-seq read

796 coverage (RPGC normalization in $1 \mathrm{~kb}$ bins with $3 \mathrm{~kb}$ smoothening). 
798 Figure S3 - (a) Boxplot displaying the composite RIP index (CRI) of C to T in CA recorded in

799 genomic windows (5 kb, $500 \mathrm{bp}$ slide), per gene, per annotated repeat, and per window

800 overlapping with the CenH3-enriched centromeres. Statistical differences for the indicated

801 comparisons were calculated using the one-sided non-parametric Mann-Whitney test; p-values <

802 0.001: ***. (b) Summary of H3K4me2 (green), H3K9me3 (red), and H3K27me3 (orange)

803 normalized ChIP-seq read coverage (RPGC normalization in $1 \mathrm{~kb}$ bins and $3 \mathrm{~kb}$ smoothening) in

804 genomic bins (2.5\%) across the chromosomal arms of the eight chromosomes of Verticillium

805 dahliae strain JR2 (divided into $2.5 \%$ bins) and the centromeric regions (divided into $10 \%$ bins).

806 The dots indicate the average ChIP-seq coverage and the whiskers indicate \pm 1.5 times the

807 interquartile range. (c-e) Boxplots displaying the (c) weighted methylation levels (CG context),

808 (d) the composite RIP index, and (e) the expression in PDB growth medium (counts per million)

809 for repetitive elements belonging to ten repeat families identified in the eight centromeres in

$810 \quad$ Verticillium dahliae JR2.

811 Figure S4 (a-c) Whole-genome alignments between the eight chromosomes of (a) Verticillium

812 dahliae strains JR2 and VdLs17 (32), (b) V. dahliae strains CQ2 and JR2 (32, 33), and (c) V.

813 dahliae strains CQ2 and VdLs17 $(32,33)$. (d-e) Schematic overview of the genome assemblies of

814 Verticillium dahliae strains (d) VdLs17 and (e) CQ2. The individual lanes show the GC content,

815 the gene (red) and repeat (blue) density (all summarized in $5 \mathrm{~kb}$ windows with $500 \mathrm{bp}$ slide), and

816 the location of the centromere associated VdLTRE9. (f) Synteny analyses of the eight

817 chromosomes of $V$. dahliae strains JR2 and CQ2. Schematic overview of the eight chromosomes

818 of $V$. dahliae strain JR2 (left) and the corresponding syntenic regions in $V$. dahliae strains CQ2

819 (right). Centromeres are indicated by stars, and syntenic centromeres of $V$. dahliae strain CQ2 are

820 colored according to Cenl-8 of V. dahliae strain JR2.

821 Figure S5- Hi-C contact matrix showing the interaction frequencies between genomic regions in

822 (a) V. nonalfalfae (T2), (b) V. alfalfae (PD683), (c) the allodiploid V. longisporum (PD589), (d) 
823 V. nubilum (397), (e) V. albo-atrum (PD747), (f) V. zaregamsianum (PD739), (g) V. tricorpus

824 (PD593), (h) V. klebhanii (PD401), and (i) V. isaacii (PD618). Regions of high inter-

825 chromosomal interaction frequencies are indicative of centromeres and are highlighted by arrow

826 heads, and the blue line indicated boundaries between the pseudo-chromosomes.

827 Figure S6 - (a-b) Comparison of normalized read coverage and corrected centromere lengths for

828 Verticillium species for which short-read data is available. (a) Counts per million mapped reads

829 (CPM) normalized read coverage was calculated for GC-biased corrected short-read libraries in

83050 bp genomic windows, excluding regions containing assembly gaps (Ns). Genomic windows

831 are summarized in boxplots (outliers not shown) by genomic location, centromeric regions (Cen,

832 blue) and non-centromeric regions (non-Cen, grey). (b) Centromeric lengths inferred by Hi-C

833 data were 'corrected' based on the ratio of normalized read depth between centromeres and non-

834 centromeric regions per chromosomes. Differences for each species compared to the overall mean

835 were computed using unpaired T-tests; p-values $<0.0001$ : ****, p-values $<0.001$ : ***, p-values

$836<0.01$ ** $^{*}$, p-values < 0.05: *. (c) The number of BLASTn matches of the VdLTRE9 consensus

837 element to the genomes of the Verticillium species separated by their genomic location,

838 centromeric regions (Cen, blue) and non-centromeric regions (non-Cen, grey). The overall

839 number of base pairs (bp) covered by the BLASTn matches in each genome sequence is

840 indicated. The asterisk denotes the high number of VdLTRE9 matches to unassigned, non-Cen

841 regions in the genome assembly of Verticillium nonalfalfae (T2). (d) The number of repetitive

842 element matches identified by RepeatMasker for each Verticillium species based on

843 species/strain-specific repeat libraries generated by RepeatModeler separated by their genomic

844 location, centromeric regions (Cen, blue) and non-centromeric regions (non-Cen, grey). (e) GC-

845 content of the Verticillium genomes in 50 bp windows and separated by their genomic location,

846 centromeric regions (Cen, blue) and non-centromeric regions (non-Cen, grey). ( $\int$ ) The repeat 
847 content of centromeric regions in percent covered sequences in the different Verticillium species.

848 Each data point summarized in the boxplot is the repeat content per centromere.

849 Figure $\mathbf{S 7}$ - Schematic overview of the centromeric regions (250 kb) in (a) Verticillium dahliae

850 strain JR2, in (b) species belonging to clade Flavnonexudans, and in (c) species belonging clade

851 Flavexudans. The centromeres are indicated by dark grey bars. The predicted genes (black) and

852 repeats (blue) are shown below each centromere, and location of VdLTRE9 (partial) matches

853 (light green) are shown above each centromere. Repeats that share sequence similarity (BLASTn)

854 to the VdLTRE9 consensus sequence are shown above each centromere (dark green).

855 Figure S8 - Sequence comparisons of de novo repeat families identified with RepeatModeler and

856 RepeatMasker in the genome assemblies of the different Verticillium species. Individual repeat

857 family consensus sequences were clustered using BLASTClust. (a) Relationships between

858 different repeat family consensus sequences are displayed as connected graphs. The sub-graph

859 with the consensus sequences with similarity to VdLTRE9 is highlighted in yellow. (b) The

860 presence/absence matrix indicates the occurrences of different repeat families in the analyzed

861 Verticillium species (black present, white absent). The cluster containing consensus sequences

862 with similarity to VdLTRE9 is highlighted.

863 Figure S9 - Reconstruction of ancestral genomes within the genus Verticillium with AnChro

864 (56). The number of (a) chromosomes and (b) genes predicted by all potential ancestral

865 reconstructions using different combinations of genomes and stringency parameters. The

866 phylogenetic tree in (a) depicts the relationships between Verticillium species and the

867 abbreviations used for the ancestors. The inlays display boxplots to summarize the number of (a)

868 chromosomes and (b) genes per ancestral reconstruction. (c) The number of chromosomes and

869 genes of the chosen 'optimal' reconstruction for each of the internal ancestors. (d) The number of

870 genes per chromosome for each of the reconstructed ancestor and the extant Verticillium species.

871 The star highlights the reconstruction for the B1 ancestor that had ten chromosomes, but with two 
872 chromosomes with six and two genes. (e) Reconstruction of the Verticillium species phylogeny

873 based on synteny relationship using PhyChro (111).

875 Table S1 - (a) Overview of the different Verticillium sequencing libraries used in this study. (b)

876 Position of the individual centromeric regions inferred by Hi-C inter-chromosomal interaction

877 frequencies and the overlap (in $\mathrm{kb}$ ) with $\mathrm{CenH} 3$-enriched regions and the centromere associated

878 VdLTRE9 in Verticillium dahliae JR2, VdLs17, and CQ2. (c) Overview of the different

879 Verticillium genomes assembled using Hi-C interactions. (d) Position, length, and number of

880 assembly gaps (Ns) of the individual centromeric regions inferred by Hi-C inter-chromosomal

881 interaction in Verticillium nonalfalfae (T2), Verticillium alfalfae (PD683), the allodiploid

882 Verticillium longisporum (PD589), Verticillium nubilum (397),Verticillium albo-atrum (PD747),

883 Verticillium zaregamsianum (PD739), Verticillium tricorpus (PD593), Verticillium klebhanii

884 (PD401), and Verticillium isaacii (PD618). (e) The number of de novo repeat consensus

885 sequences identified within and outside of centromeric regions in the Verticillium species. Only

886 consensus elements with $>5$ matches in centromeric regions are displayed. Note that the

887 consensus names between species/strains are not comparable. (f) The primers used for cloning the

888 CenH3 FLAG tag in Verticillium dahliae strain JR2 


\section{REFERENCES}

891 1. Roy B, Sanyal K. 2011. Diversity in requirement of genetic and epigenetic factors for

892 centromere function in fungi. Eukaryotic cell 10:1384-1395.

893 2. Foley EA, Kapoor TM. 2013. Microtubule attachment and spindle assembly checkpoint

894 signalling at the kinetochore. Nature reviews Molecular cell biology 14:25-37.

895 3. Burrack LS, Berman J. 2012. Flexibility of centromere and kinetochore structures. Trends 896 in Genetics 28:204-212.

897 4. Janssen A, van der Burg M, Szuhai K, Kops GJPL, Medema RH. 2011. Chromosome 898 segregation errors as a cause of DNA damage and structural chromosome aberrations. Science 899 (New York, NY) 333:1895-1898.

900 5. Sheltzer JM, Blank HM, Pfau SJ, Tange Y, George BM, Humpton TJ, Brito IL, Hiraoka 901 Y, Niwa O, Amon A. 2011. Aneuploidy drives genomic instability in yeast. Science (New York, $902 N Y)$ 333:1026-1030.

903 6. Barra V, Fachinetti D. 2018. The dark side of centromeres: Types, causes and 904 consequences of structural abnormalities implicating centromeric DNA. Nature Communications $9059: 4340$.

906 7. Seidl MF, Thomma BPHJ. 2014. Sex or no sex: Evolutionary adaptation occurs 907 regardless. BioEssays 36:335-345.

908 8. Faino L, Seidl MF, Shi-Kunne X, Pauper M, van den Berg GCM, Wittenberg AHJ, 909 Thomma BPHJ. 2016. Transposons passively and actively contribute to evolution of the two910 speed genome of a fungal pathogen. Genome Research 26:1091-1100.

911 9. Rancati G, Pavelka N, Fleharty B, Noll A, Trimble R, Walton K, Perera A, Staehling912 Hampton K, Seidel CW, Li R. 2008. Aneuploidy underlies rapid adaptive evolution of yeast cells 913 deprived of a conserved cytokinesis motor. Cell 135:879-893.

914 10. Pavelka N, Rancati G, Zhu J, Bradford WD, Saraf A, Florens L, Sanderson BW, Hattem 915 GL, Li R. 2010. Aneuploidy confers quantitative proteome changes and phenotypic variation in 916 budding yeast. Nature 468:321-325. 
917 11. Sankaranarayanan SR, Ianiri G, Coelho MA, Reza MH, Thimmappa BC, Ganguly P,

918 Vadnala RN, Sun S, Siddharthan R, Tellgren-Roth C, Dawson TLJ, Heitman J, Sanyal K. 2020.

919 Loss of centromere function drives karyotype evolution in closely related Malassezia species.

920 Elife 9.

921 12. Ola M, O'Brien CE, Coughlan AY, Ma Q, Donovan PD, Wolfe KH, Butler G. 2020.

922 Polymorphic centromere locations in the pathogenic yeast Candida parapsilosis. bioRxiv 923 doi:10.1101/2020.04.09.034512:2020.04.09.034512.

924 13. Yadav V, Sreekumar L, Guin K, Sanyal K. 2018. Five pillars of centromeric chromatin in 925 fungal pathogens. PLoS pathogens 14:e1007150.

926 14. Henikoff S, Ahmad K, Malik HS. 2001. The centromere paradox: Stable inheritance with 927 rapidly evolving DNA. Science 293:1098-102.

928 15. Smith KM, Galazka JM, Phatale PA, Connolly LR, Freitag M. 2012. Centromeres of 929 filamentous fungi. Chromosome research : an international journal on the molecular, 930 supramolecular and evolutionary aspects of chromosome biology 20:635-656.

931 16. Yadav V, Sun S, Billmyre RB, Thimmappa BC, Shea T, Lintner R, Bakkeren G, Cuomo 932 CA, Heitman J, Sanyal K. 2018. RNAi is a critical determinant of centromere evolution in closely 933 related fungi. Proceedings of the National Academy of Sciences of the United States of America 934 115:3108-3113.

935 17. Fitzgerald-Hayes M, Clarke L, Carbon J. 1982. Nucleotide sequence comparisons and 936 functional analysis of yeast centromere DNAs. Cell 29:235-244.

937 18. Furuyama S, Biggins S. 2007. Centromere identity is specified by a single centromeric 938 nucleosome in budding yeast. Proceedings of the National Academy of Sciences of the United 939 States of America 104:14706-14711.

940 19. Krassovsky K, Henikoff JG, Henikoff S. 2012. Tripartite organization of centromeric 941 chromatin in budding yeast. Proceedings of the National Academy of Sciences of the United 942 States of America 109:243-248.

943 20. Cliften PF, Fulton RS, Wilson RK, Johnston M. 2006. After the duplication: gene loss 944 and adaptation in Saccharomyces genomes. Genetics 172:863-872. 
945 21. Baum M, Sanyal K, Mishra PK, Thaler N, Carbon J. 2006. Formation of functional 946 centromeric chromatin is specified epigenetically in Candida albicans. Proceedings of the

947 National Academy of Sciences of the United States of America 103:14877-14882.

948 22. Padmanabhan S, Thakur J, Siddharthan R, Sanyal K. 2008. Rapid evolution of Cse4p-rich 949 centromeric DNA sequences in closely related pathogenic yeasts, Candida albicans and Candida 950 dubliniensis. Proceedings of the National Academy of Sciences of the United States of America $951 \quad 105: 19797-19802$.

952 23. Sanyal K, Baum M, Carbon J. 2004. Centromeric DNA sequences in the pathogenic yeast 953 Candida albicans are all different and unique. Proceedings of the National Academy of Sciences 954 of the United States of America 101:11374-11379.

955 24. Cambareri EB, Aisner R, Carbon J. 1998. Structure of the chromosome VII centromere 956 region in Neurospora crassa: degenerate transposons and simple repeats. Molecular and cellular 957 biology 18:5465-5477.

958 25. Smith KM, Phatale PA, Sullivan CM, Pomraning KR, Freitag M. 2011. Heterochromatin 959 is required for normal distribution of Neurospora crassa CenH3. Molecular and cellular biology $960 \quad 31: 2528-2542$.

961 26. Selker EU. 2002. Repeat-induced gene silencing in fungi. Advances in genetics 46:439962450.

963 27. Schotanus K, Soyer JL, Connolly LR, Grandaubert J, Happel P, Smith KM, Freitag M, 964 Stukenbrock EH. 2015. Histone modifications rather than the novel regional centromeres of 965 Zymoseptoria tritici distinguish core and accessory chromosomes. Epigenetics \& Chromatin 8:41.

966 28. Thomma BPHJ, Seidl MF, Shi-Kunne X, Cook DE, Bolton MD, van Kan JAL, Faino L. 967 2016. Mind the gap; Seven reasons to close fragmented genome assemblies. Fungal Genetics and 968 Biology 90:24-30.

969 29. Yadav V, Yang F, Reza MH, Liu S, Valent B, Sanyal K, Naqvi NI. 2019. Cellular 970 dynamics and genomic identity of centromeres in cereal blast fungus. mBio 10.

971 30. Fang Y, Coelho MA, Shu H, Schotanus K, Thimmappa BC, Yadav V, Chen H, Malc EP, 972 Wang J, Mieczkowski PA, Kronmiller B, Tyler BM, Sanyal K, Dong S, Nowrousian M, Heitman 
973 J. 2020. Long transposon-rich centromeres in an oomycete reveal divergence of centromere

974 features in Stramenopila-Alveolata-Rhizaria lineages. PLoS Genetics 16:e1008646.

975 31. Navarro-Mendoza MI, Perez-Arques C, Panchal S, Nicolas FE, Mondo SJ, Ganguly P,

976 Pangilinan J, Grigoriev IV, Heitman J, Sanyal K, Garre V. 2019. Early diverging fungus Mucor

977 circinelloides lacks centromeric histone CENP-A and displays a mosaic of point and regional

978 centromeres. Current Biology 29:3791-3802 e6.

979 32. Faino L, Seidl MF, Datema E, van den Berg GCM, Janssen A, Wittenberg AHJ, Thomma

980 BPHJ. 2015. Single-Molecule Real-Time sequencing combined with optical mapping yields

981 completely finished fungal genome. mBio 6:e00936-15.

982 33. Depotter JRL, Shi-Kunne X, Missonnier H, Liu T, Faino L, van den Berg GCM, Wood

983 TA, Zhang B, Jacques A, Seidl MF, Thomma BPHJ. 2019. Dynamic virulence-related regions of

984 the plant pathogenic fungus Verticillium dahliae display enhanced sequence conservation.

985 Molecular Ecology 28:3482-3495.

986 34. de Jonge R, van Esse PH, Maruthachalam K, Bolton MD, Santhanam P, Saber MK,

987 Zhang Z, Usami T, Lievens B, Subbarao KV, Thomma BPHJ. 2012. Tomato immune receptor

988 Ve1 recognizes effector of multiple fungal pathogens uncovered by genome and RNA

989 sequencing. Proceedings of the National Academy of Sciences of the United States of America

990 109:5110-5115.

991 35. de Jonge R, Bolton MD, Kombrink A, van den Berg GCM, Yadeta KA, Thomma BPHJ.

992 2013. Extensive chromosomal reshuffling drives evolution of virulence in an asexual pathogen.

993 Genome research 23:1271-1282.

994 36. Cook DE, Kramer M, Seidl MF, Thomma BP. 2020. Chromatin features define adaptive

995 genomic regions in a fungal plant pathogen. bioRxiv

996 doi:10.1101/2020.01.27.921486:2020.01.27.921486.

997 37. Inderbitzin P, Bostock RM, Davis RM, Usami T, Platt HW, Subbarao KV. 2011.

998 Phylogenetics and taxonomy of the fungal vascular wilt pathogen Verticillium, with the 999 descriptions of five new species. PloS one 6:e28341.

1000 38. Inderbitzin P, Davis RM, Bostock RM, Subbarao KV. 2011. The ascomycete Verticillium

1001 longisporum is a hybrid and a plant pathogen with an expanded host range. PloS one 6:e18260. 
39. Depotter JRL, Beveren Fv, Rodriguez-Moreno L, van den Berg GCM, Wood TA, Thomma BPHJ, Seidl MF. 2018. Homogenization of sub-genome secretome gene expression

1004 patterns in the allodiploid fungus Verticillium longisporum. bioRxiv:341636.

1005 40. Shi-Kunne X, Faino L, van den Berg GCM, Thomma BPHJ, Seidl MF. 2018. Evolution 1006 within the fungal genus Verticillium is characterized by chromosomal rearrangement and gene 1007 loss. Environmental Microbiology 20:1362-1373.

1008 41. Depotter JRL, Seidl MF, van den Berg GCM, Thomma BPHJ, Wood TA. 2017. A 1009 distinct and genetically diverse lineage of the hybrid fungal pathogen Verticillium longisporum 1010 population causes stem striping in British oilseed rape. Environmental Microbiology 19:399710114009.

1012 42. Moeller M, Habig M, Lorrain C, Feurtey A, Haueisen J, Fagundes WC, Alizadeh A, 1013 Freitag M, Stukenbrock EH. 2020. Recent loss of the Dim2 cytosine DNA methyltransferase 1014 impacts mutation rate and evolution in a fungal plant pathogen. bioRxiv 1015 doi:10.1101/2020.03.27.012203:2020.03.27.012203.

1016 43. Klosterman SJ, Subbarao KV, Kang S, Veronese P, Gold SE, Thomma BPHJ, Chen Z, 1017 Henrissat B, Lee Y-H, Park J, Garcia-Pedrajas MD, Barbara DJ, Anchieta A, de Jonge R, 1018 Santhanam P, Maruthachalam K, Atallah Z, Amyotte SG, Paz Z, Inderbitzin P, Hayes RJ, Heiman 1019 DI, Young S, Zeng Q, Engels R, Galagan J, Cuomo CA, Dobinson KF, Ma L-J. 2011. 1020 Comparative genomics yields insights into niche adaptation of plant vascular wilt pathogens. $1021 \quad$ PLoS pathogens 7:e1002137.

1022 44. Amyotte SG, Tan X, Pennerman K, del Mar Jimenez-Gasco M, Klosterman SJ, Ma L-J, 1023 Dobinson KF, Veronese P. 2012. Transposable elements in phytopathogenic Verticillium spp.: 1024 insights into genome evolution and inter- and intra-specific diversification. BMC genomics $1025 \quad 13: 314$.

1026 45. Lewis ZA, Honda S, Khlafallah TK, Jeffress JK, Freitag M, Mohn F, Schubeler D, Selker 1027 EU. 2009. Relics of repeat-induced point mutation direct heterochromatin formation in 1028 Neurospora crassa. Genome Research 19:427-437.

1029 46. Liu S-Y, Lin J-Q, Wu H-L, Wang C-C, Huang S-J, Luo Y-F, Sun J-H, Zhou J-X, Yan S1030 J, He J-G, Wang J, He Z-M. 2012. Bisulfite sequencing reveals that Aspergillus flavus holds a 1031 hollow in DNA methylation. PloS one 7:e30349. 
1032 47. Seymour M, Ji L, Santos AM, Kamei M, Sasaki T, Basenko EY, Schmitz RJ, Zhang X,

1033 Lewis ZA. 2016. Histone H1 Limits DNA Methylation in Neurospora crassa. G3 6:1879-1889.

1034 48. Kursel LE, Malik HS. 2016. Centromeres. Current Biology 26:R487-R490.

1035 49. Friedman S, Freitag M. 2017. Evolving centromeres and kinetochores. Advances in 1036 Genetics 98:1-41.

1037 50. Flutre T, Duprat E, Feuillet C, Quesneville H. 2011. Considering transposable element 1038 diversification in de novo annotation approaches. PloS one 6:e16526.

1039 51. Galazka JM, Klocko AD, Uesake M, Honda S, Selker EU, Freitag M. 2016. Neurospora 1040 chromosomes are organized by blocs of importin alpha-dependent heterochromatin that are 1041 largely independent of H3K9me3. Genome research doi:10.1101/gr.203182.115:gr.203182.115.

1042 52. Winter DJ, Ganley ARD, Young CA, Liachko I, Schardl CL, Dupont P-y, Berry D, Ram 1043 A, Scott B, Cox MP. 2018. Repeat elements organise 3D genome structure and mediate 1044 transcription in the filamentous fungus Epichloë festucae. PLoS Genetics 14:e1007467.

1045 53. Marie-Nelly H, Marbouty M, Cournac A, Flot J-F, Liti G, Parodi DP, Syan S, Guillén N, 1046 Margeot A, Zimmer C, Koszul R. 2014. High-quality genome (re)assembly using chromosomal 1047 contact data. Nature Communications 5:5695.

1048 54. Mizuguchi T, Fudenberg G, Mehta S, Belton J-M, Taneja N, Folco HD, FitzGerald P, 1049 Dekker J, Mirny L, Barrowman J, Grewal SIS. 2014. Cohesin-dependent globules and 1050 heterochromatin shape 3D genome architecture in S. pombe. Nature 516:432-435.

1051 55. Varoquaux N, Liachko I, Ay F, Burton JN, Shendure J, Dunham MJ, Vert J-P, Noble 1052 WS. 2015. Accurate identification of centromere locations in yeast genomes using Hi-C. Nucleic 1053 Acids Research 43:5331-5339.

1054 56. Vakirlis N, Sarilar V, Drillon G, Fleiss A, Agier N, Meyniel JP, Blanpain L, Carbone A, 1055 Devillers H, Dubois K, Gillet-Markowska A, Graziani S, Huu-Vang N, Poirel M, Reisser C, 1056 Schott J, Schacherer J, Lafontaine I, Llorente B, Neuveglise C, Fischer G. 2016. Reconstruction 1057 of ancestral chromosome architecture and gene repertoire reveals principles of genome evolution 1058 in a model yeast genus. Genome Research 26:918-32. 
1059 57. Depotter JR, Deketelaere S, Inderbitzin P, Tiedemann AV, Hofte M, Subbarao KV, 1060 Wood TA, Thomma BP. 2016. Verticillium longisporum, the invisible threat to oilseed rape and 1061 other brassicaceous plant hosts. Molecular Plant Pathology 17:1004-16.

1062 58. Muller H, Gil J, Jr., Drinnenberg IA. 2019. The impact of centromeres on spatial genome 1063 architecture. Trends in Genetics 35:565-578.

1064 59. Treangen TJ, Salzberg SL. 2011. Repetitive DNA and next-generation sequencing: 1065 Computational challenges and solutions. Nature Reviews Genetics 13:36-46.

1066 60. Seidl MF, Faino L, Shi-Kunne X, van den Berg GCM, Bolton MD, Thomma BPHJ. 1067 2015. The genome of the saprophytic fungus Verticillium tricorpus reveals a complex effector 1068 repertoire resembling that of its pathogenic relatives. Molecular Plant-Microbe Interactions 1069 28:362-373.

1070 61. Sun S, Yadav V, Billmyre RB, Cuomo CA, Nowrousian M, Wang L, Souciet JL, 1071 Boekhout T, Porcel B, Wincker P, Granek JA, Sanyal K, Heitman J. 2017. Fungal genome and 1072 mating system transitions facilitated by chromosomal translocations involving intercentromeric 1073 recombination. PLoS Biology 15:e2002527.

1074 62. International Chicken Genome Sequencing C. 2004. Sequence and comparative analysis 1075 of the chicken genome provide unique perspectives on vertebrate evolution. Nature 432:695-716.

1076 63. Fukagawa T. 2017. Critical histone post-translational modifications for centromere 1077 function and propagation. Cell Cycle 16:1259-1265.

1078 64. Sullivan BA, Karpen GH. 2004. Centromeric chromatin exhibits a histone modification 1079 pattern that is distinct from both euchromatin and heterochromatin. Nature Structural \& 1080 Molecular Biology 11:1076-83.

1081 65. Volpe TA, Kidner C, Hall IM, Teng G, Grewal SI, Martienssen RA. 2002. Regulation of 1082 heterochromatic silencing and histone H3 lysine-9 methylation by RNAi. Science 297:1833-7.

1083 66. Li X, Wang X, He K, Ma Y, Su N, He H, Stolc V, Tongprasit W, Jin W, Jiang J, 1084 Terzaghi W, Li S, Deng XW. 2008. High-resolution mapping of epigenetic modifications of the 1085 rice genome uncovers interplay between DNA methylation, histone methylation, and gene 1086 expression. Plant Cell 20:259-76. 
1087 67. Blower MD, Sullivan BA, Karpen GH. 2002. Conserved organization of centromeric 1088 chromatin in flies and humans. Dev Cell 2:319-30.

1089 68. Lewis ZA, Adhvaryu KK, Honda S, Shiver AL, Knip M, Sack R, Selker EU. 2010. DNA 1090 methylation and normal chromosome behavior in Neurospora depend on five components of a 1091 histone methyltransferase complex, DCDC. PLoS Genetics 6:e1001196.

1092 69. Bayne EH, White SA, Kagansky A, Bijos DA, Sanchez-Pulido L, Hoe KL, Kim DU, 1093 Park HO, Ponting CP, Rappsilber J, Allshire RC. 2010. Stc1: A critical link between RNAi and 1094 chromatin modification required for heterochromatin integrity. Cell 140:666-77.

1095 70. Buhler M, Moazed D. 2007. Transcription and RNAi in heterochromatic gene silencing. 1096 Nature Structural \& Molecular Biology 14:1041-8.

1097 71. Yang J, Sun S, Zhang S, Gonzalez M, Dong Q, Chi Z, Chen YH, Li F. 2018. 1098 Heterochromatin and RNAi regulate centromeres by protecting CENP-A from ubiquitin-mediated 1099 degradation. PLoS Genetics 14:e1007572.

1100 72. Kagansky A, Folco HD, Almeida R, Pidoux AL, Boukaba A, Simmer F, Urano T, 1101 Hamilton GL, Allshire RC. 2009. Synthetic heterochromatin bypasses RNAi and centromeric 1102 repeats to establish functional centromeres. Science 324:1716-9.

1103 73. Jesenicnik T, Stajner N, Radisek S, Jakse J. 2019. RNA interference core components 1104 identified and characterised in Verticillium nonalfalfae, a vascular wilt pathogenic plant fungi of 1105 hops. Scientific Reports 9:8651.

1106 74. Altschul SF, Gish W, Miller W, Myers EW, Lipman DJ. 1990. Basic local alignment 1107 search tool. Journal of molecular biology 215:403-410.

1108 75. Camacho C, Coulouris G, Avagyan V, Ma N, Papadopoulos J, Bealer K, Madden TL. 1109 2009. BLAST+: architecture and applications. BMC Bioinformatics 10:421.

1110 76. Slater GS, Birney E. 2005. Automated generation of heuristics for biological sequence 1111 comparison. BMC Bioinformatics 6:31.

1112 77. Katoh K, Standley DM. 2013. MAFFT multiple sequence alignment software version 7: 1113 improvements in performance and usability. Molecular biology and evolution 30:772-780. 
1114 78. Nguyen LT, Schmidt HA, von Haeseler A, Minh BQ. 2015. IQ-TREE: A fast and 1115 effective stochastic algorithm for estimating maximum-likelihood phylogenies. Molecular 1116 Biology and Evolution 32:268-74.

1117 79. Frandsen RJ, Andersson JA, Kristensen MB, Giese H. 2008. Efficient four fragment 1118 cloning for the construction of vectors for targeted gene replacement in filamentous fungi. $B M C$ 1119 Mol Biol 9:70.

1120 80. Santhanam P. 2012. Random insertional mutagenesis in fungal genomes to identify 1121 virulence factors. Methods Mol Biol 835:509-17.

1122 81. Bolger AM, Lohse M, Usadel B. 2014. Trimmomatic: a flexible trimmer for Illumina 1123 sequence data. Bioinformatics (Oxford, England) 30:2114-2120.

1124 82. Langmead B, Salzberg SL. 2012. Fast gapped-read alignment with Bowtie 2. Nature 1125 methods 9:357-359.

1126 83. Li H, Durbin R. 2009. Fast and accurate short read alignment with Burrows-Wheeler 1127 transform. Bioinformatics (Oxford, England) 25:1754-1760.

1128 84. Ramirez F, Ryan DP, Gruning B, Bhardwaj V, Kilpert F, Richter AS, Heyne S, Dundar F, 1129 Manke T. 2016. deepTools2: a next generation web server for deep-sequencing data analysis. $1130 \quad$ Nucleic Acids Res 44:W160-5.

1131 85. Zhang Y, Liu T, Meyer CA, Eeckhoute J, Johnson DS, Bernstein BE, Nusbaum C, Myers 1132 RM, Brown M, Li W, Liu XS. 2008. Model-based analysis of ChIP-Seq (MACS). Genome 1133 Biology 9:R137.

1134 86. Schultz MD, Schmitz RJ, Ecker JR. 2012. 'Leveling' the playing field for analyses of 1135 single-base resolution DNA methylomes. Trends in Genetics 28:583-585.

1136 87. Durand NC, Shamim MS, Machol I, Rao SS, Huntley MH, Lander ES, Aiden EL. 2016. 1137 Juicer Provides a One-Click System for Analyzing Loop-Resolution Hi-C Experiments. Cell 1138 Systems 3:95-8.

1139 88. Dudchenko O, Batra SS, Omer AD, Nyquist SK, Hoeger M, Durand NC, Shamim MS, 1140 Machol I, Lander ES, Aiden AP, Aiden EL. 2017. De novo assembly of the Aedes aegypti 1141 genome using Hi-C yields chromosome-length scaffolds. Science 356:92-95. 
1142 89. Dudchenko O, Shamim MS, Batra SS, Durand NC, Musial NT, Mostofa R, Pham M, 1143 Glenn St Hilaire B, Yao W, Stamenova E, Hoeger M, Nyquist SK, Korchina V, Pletch K, 1144 Flanagan JP, Tomaszewicz A, McAloose D, Pérez Estrada C, Novak BJ, Omer AD, Aiden EL. 1145 2018. The Juicebox Assembly Tools module facilitates de novo assembly of mammalian genomes 1146 with chromosome-length scaffolds for under \$1000. bioRxiv doi:10.1101/254797:254797.

1147 90. Jakse J, Jelen V, Radisek S, de Jonge R, Mandelc S, Majer A, Curk T, Zupan B, Thomma 1148 B, Javornik B. 2018. Genome sequence of a lethal strain of xylem-Invading Verticillium 1149 nonalfalfae. Genome Announcements 6.

1150 91. de Jonge R, Peter van Esse H, Maruthachalam K, Bolton MD, Santhanam P, Saber MK, 1151 Zhang Z, Usami T, Lievens B, Subbarao KV, Thomma BPHJ. 2012. Tomato immune receptor 1152 Ve1 recognizes effector of multiple fungal pathogens uncovered by genome and RNA 1153 sequencing. Proceedings of the National Academy of Sciences of the United States of America 1154 109:5110-5115.

1155 92. Quinlan AR, Hall IM. 2010. BEDTools: A flexible suite of utilities for comparing 1156 genomic features. Bioinformatics 26:841-2.

1157 93. Benjamini Y, Speed TP. 2012. Summarizing and correcting the GC content bias in high1158 throughput sequencing. Nucleic Acids Research 40:e72.

1159 94. Dobin A, Gingeras TR. 2015. Mapping RNA-seq reads with STAR. Current Protocols in 1160 Bioinformatics 51:11 14 1-11 14 19.

1161 95. Lawrence M, Huber W, Pages H, Aboyoun P, Carlson M, Gentleman R, Morgan MT, 1162 Carey VJ. 2013. Software for computing and annotating genomic ranges. PLoS Computational 1163 Biology 9:e1003118.

1164 96. Ellinghaus D, Kurtz S, Willhoeft U. 2008. LTRharvest, an efficient and flexible software 1165 for de novo detection of LTR retrotransposons. BMC Bioinformatics 9:18.

1166 97. Steinbiss S, Willhoeft U, Gremme G, Kurtz S. 2009. Fine-grained annotation and 1167 classification of de novo predicted LTR retrotransposons. Nucleic Acids Res 37:7002-13.

1168 98. Hoede C, Arnoux S, Moisset M, Chaumier T, Inizan O, Jamilloux V, Quesneville H. 1169 2014. PASTEC: an automatic transposable element classification tool. PloS one 9:e91929. 
1170 99. Bailly-Bechet M, Haudry A, Lerat E. 2014. "One code to find them all": a perl tool to

1171 conveniently parse RepeatMasker output files. Mobile DNA 5:13.

1172 100. Palmer JM, JE S. 2016. Funannotate: Eukaryotic genome annotation pipeline.

1173 http://funannotate.readthedocs.io. Accessed

1174 101. Cook DE, Valle-Inclan JE, Pajoro A, Rovenich H, Thomma B, Faino L. 2019. Long-

1175 Read Annotation: Automated Eukaryotic Genome Annotation Based on Long-Read cDNA

1176 Sequencing. Plant Physiol 179:38-54.

1177 102. Haas BJ, Salzberg SL, Zhu W, Pertea M, Allen JE, Orvis J, White O, Buell CR, Wortman

1178 JR. 2008. Automated eukaryotic gene structure annotation using EVidenceModeler and the 1179 Program to Assemble Spliced Alignments. Genome Biology 9:R7.

1180 103. Shannon P, Markiel A, Ozier O, Baliga NS, Wang JT, Ramage D, Amin N, Schwikowski 1181 B, Ideker T. 2003. Cytoscape: A software environment for integrated models of biomolecular 1182 interaction networks. Genome Research 13:2498-504.

1183 104. Team RC. 2013. A Language and Environment for Statistical Computing\}.

1184 105. Wickham H. 2016. ggplot2: Elegant Graphics for Data Analysis \}.

1185 106. Gel B, Serra E. 2017. karyoploteR: an R/Bioconductor package to plot customizable 1186 genomes displaying arbitrary data. Bioinformatics 33:3088-3090.

1187 107. Hahne F, Ivanek R. 2016. Visualizing genomic data using Gviz and Bioconductor. 1188 Methods in molecular biology (Clifton, NJ) 1418:335-351.

1189 108. Sullivan MJ, Petty NK, Beatson SA. 2011. Easyfig: A genome comparison visualizer. 1190 Bioinformatics 27:1009-10.

1191 109. Kurtz S, Phillippy A, Delcher AL, Smoot M, Shumway M, Antonescu C, Salzberg SL. 1192 2004. Versatile and open software for comparing large genomes. Genome Biology 5:R12.

1193 110. Drillon G, Carbone A, Fischer G. 2014. SynChro: A fast and easy tool to reconstruct and 1194 visualize synteny blocks along eukaryotic chromosomes. PLoS One 9:e92621. 
bioRxiv preprint doi: https://doi.org/10.1101/2020.06 29.179234; this version posted June 30, 2020. The copyright holder for this preprint (which was not certified by peer review) is the author/funder, who has granted bioRxiv a license to display the preprint in perpetuity. It is made available under aCC-BY-ND 4.0 International license.

1195 111. Drillon G, Champeimont R, Oteri F, Fischer G, Carbone A. 2020. Phylogenetic 1196 reconstruction based on synteny block and gene adjacencies. Molecular Biology and Evolution 1197 doi:10.1093/molbev/msaa114.

1198 
bioRxiv preprint doi: https://doi.org/10.1101/2020.06.29.179234; this version posted June 30,2020 . The copyright holder for this preprint (which was not certified by peer review) is the author/funder, who has granted bioRxiv a license to display the preprint in perpetuity. It is made available under aCC-BY-ND 4.0 International license.
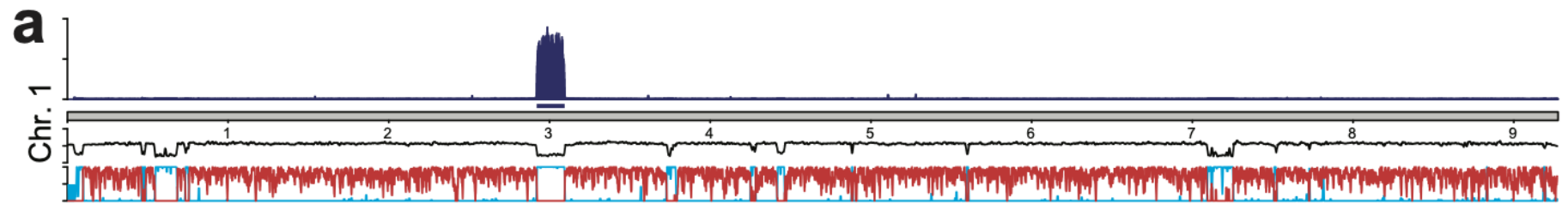

CenH3 $(0-300)$

CenH3 enrichment

GC-content (0 - 100)

Genes (I, 0 - 1)

Repeats (I, 0 - 1)

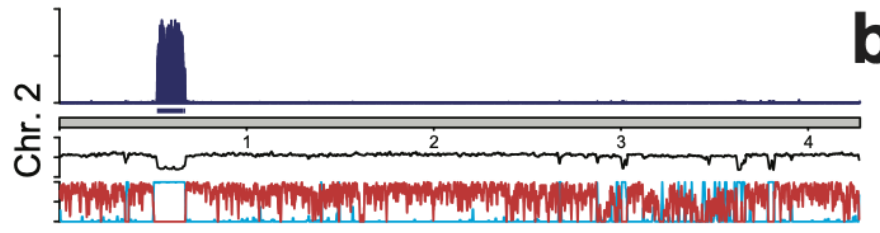

b
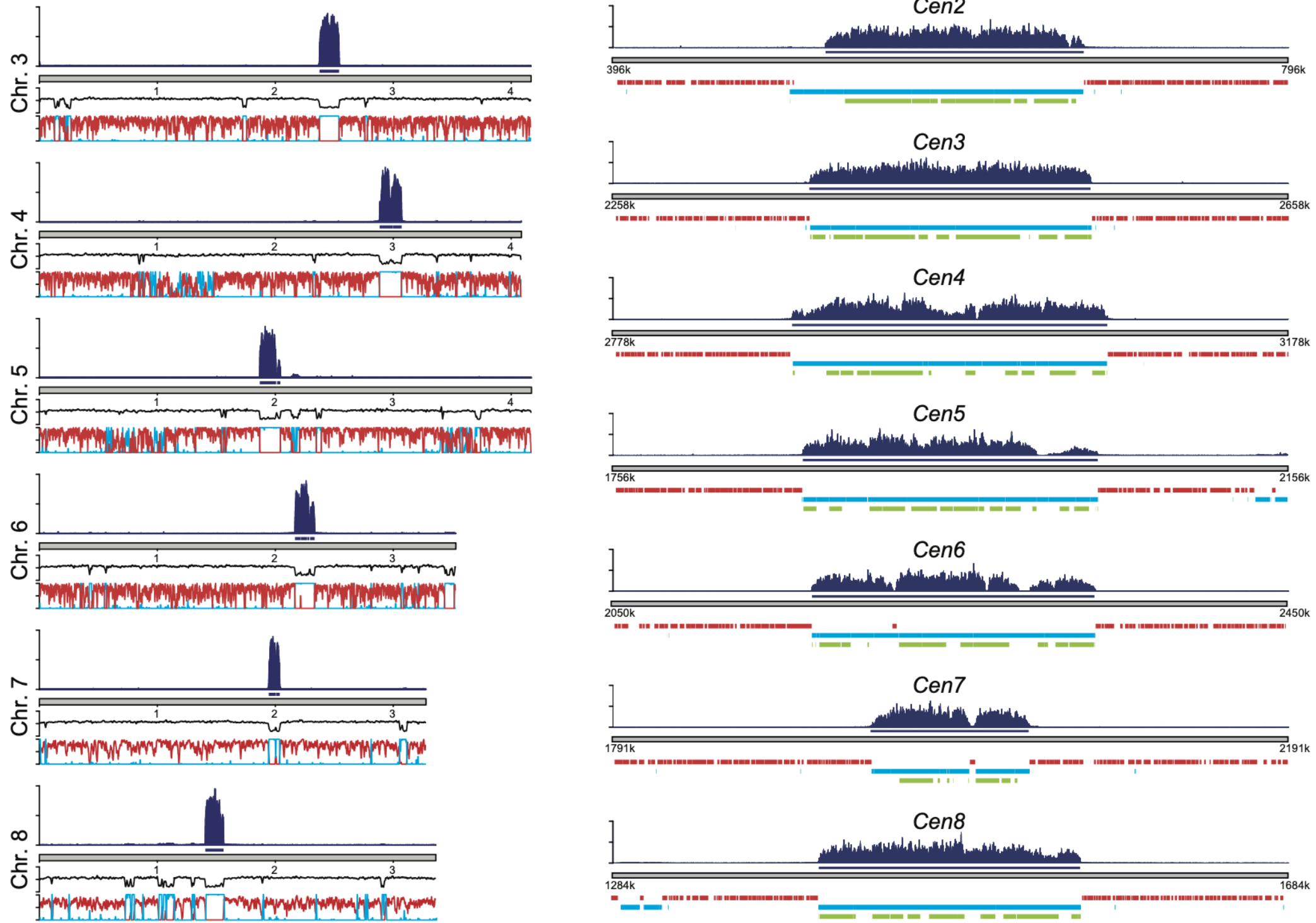


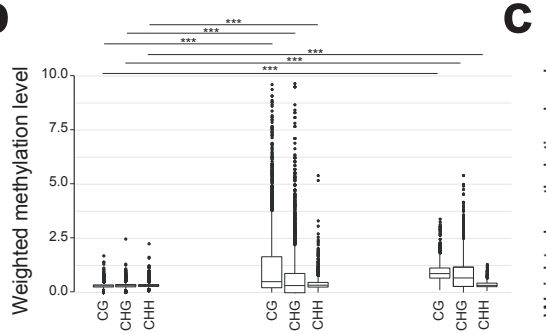

Genes

$(n=11,426)$

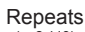

$(n=2,113)$
Centromere

$(n=2,621)$

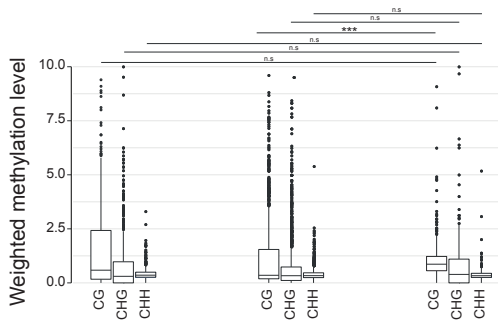

Sub-telomere Chromosome arm Centromere $(n=483)$ $(n=1,261)$

$(n=369)$

e

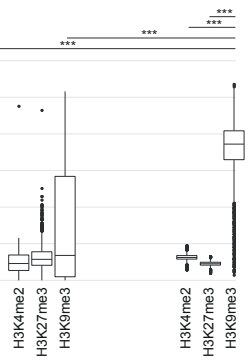

Repeats
Centromere $(n=2,621)$

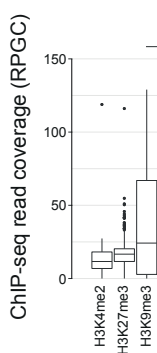

Sub-telomere $(n=483)$

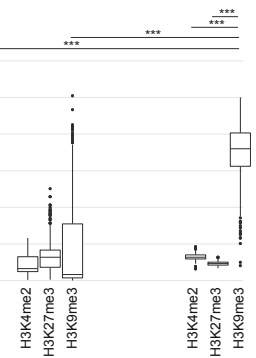

Chromosome arm $(n=1,261)$
Centromere $(n=369)$ 


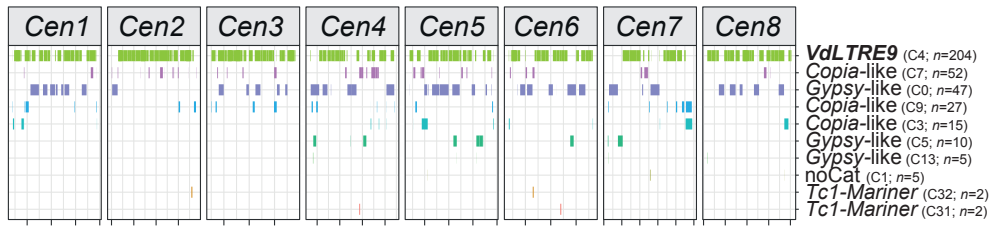

Chr. 2 tiun

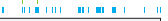

\section{Cen3}

Chr. 3

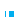
:H.1.

Cen4

Chr. 4

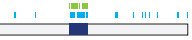

Cen5

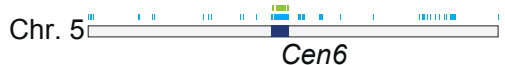

Chr. 6rint

Chr. $7 \stackrel{\text { Cen } 8}{\longleftarrow}$

Chr. 8

\section{Distribution of centromere repeats}

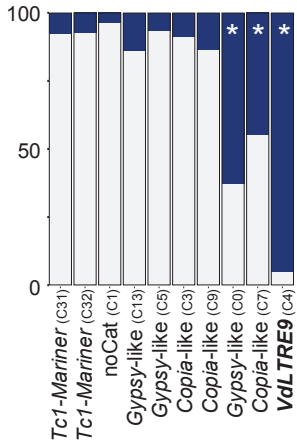

- Cen

non-Cen 
a Verticillium dahliae JR2

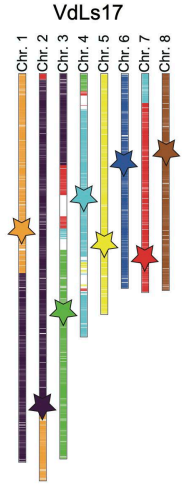

b

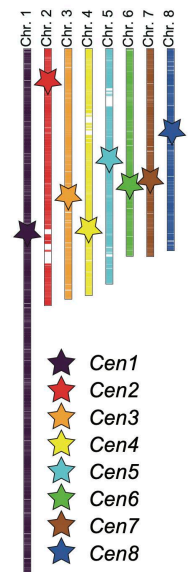

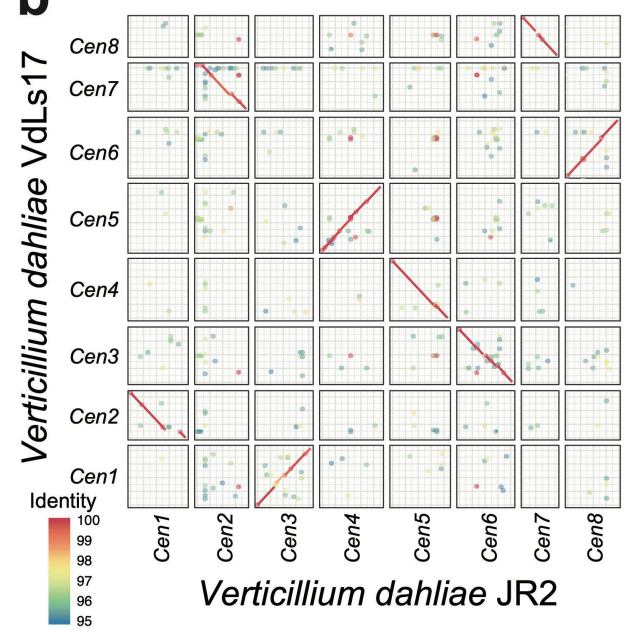

C Verticillium dahliae JR2

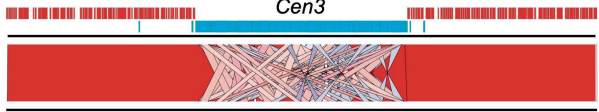

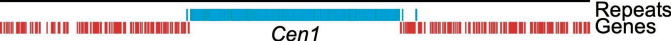
Verticillium dahliae VdLs17 $84 \% \frac{\text { Direct }}{\text { Inversion }} 100 \%$

d

f

e

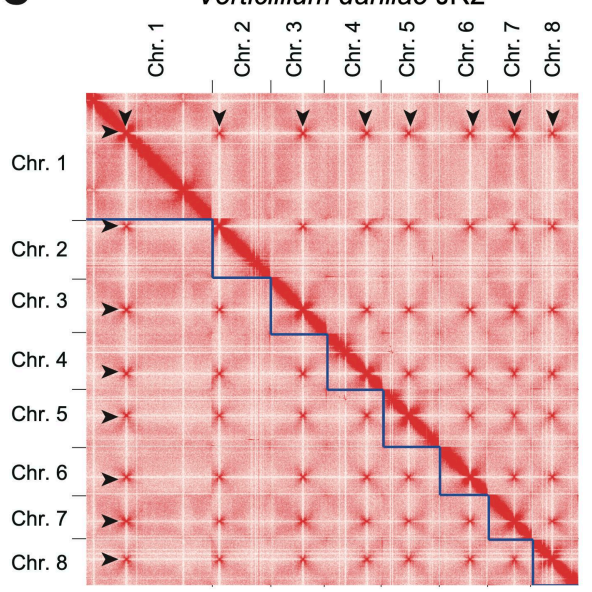

Verticillium dahliae VdLs17

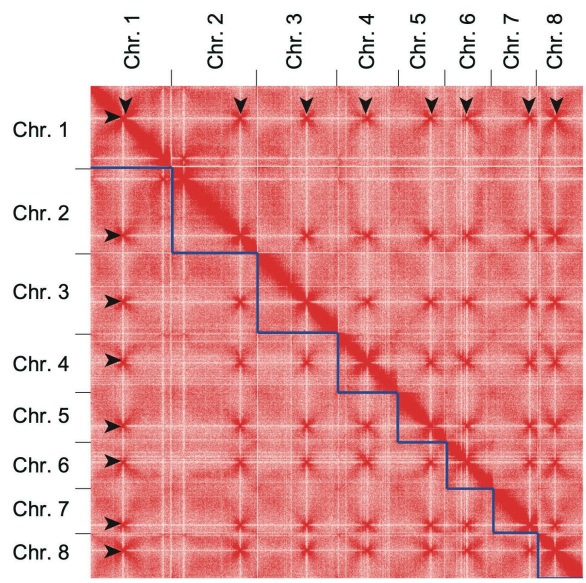

Distribution of centromere repeats

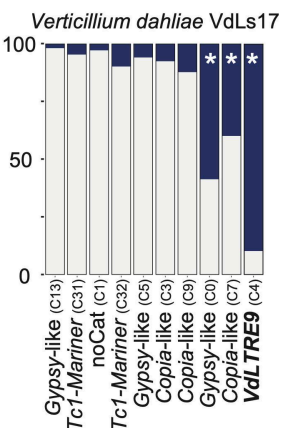

Verticillium dahliae CQ2
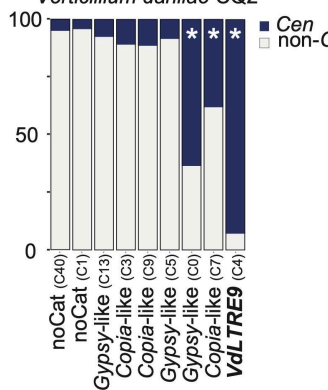

Verticillium dahliae CQ2

9

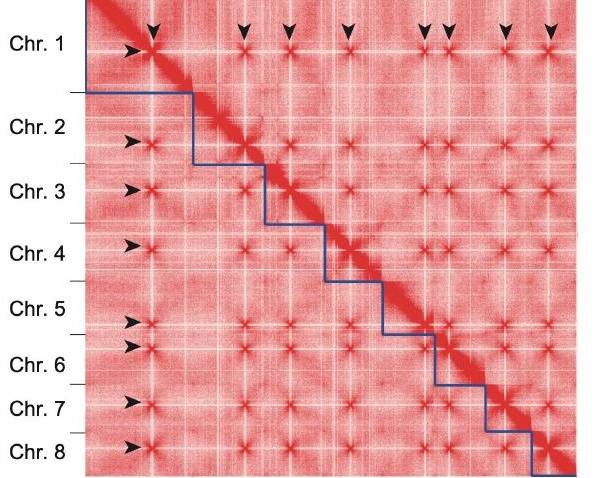


a

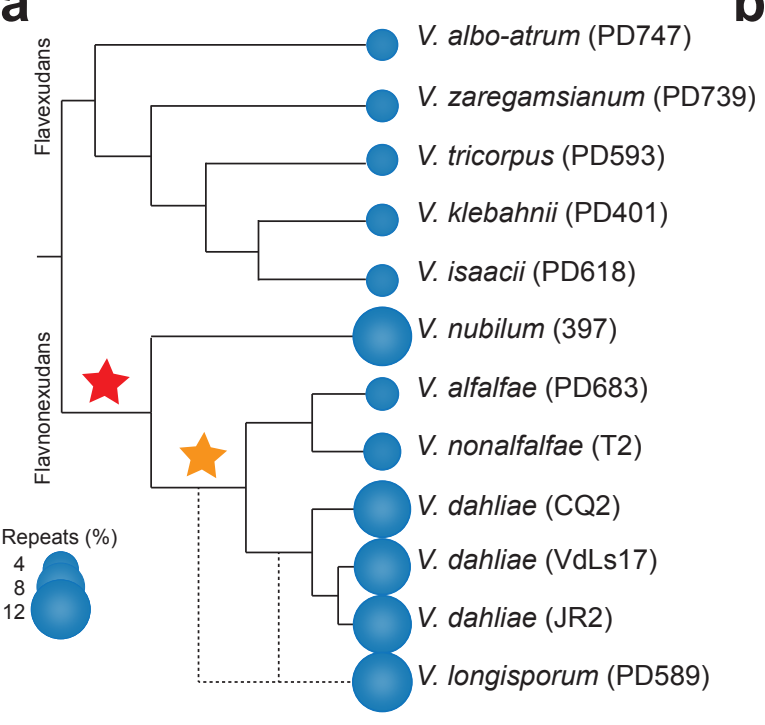

b ANOVA, $p<2.2 \mathrm{e}-16$

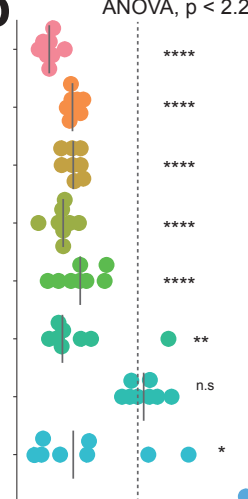

e

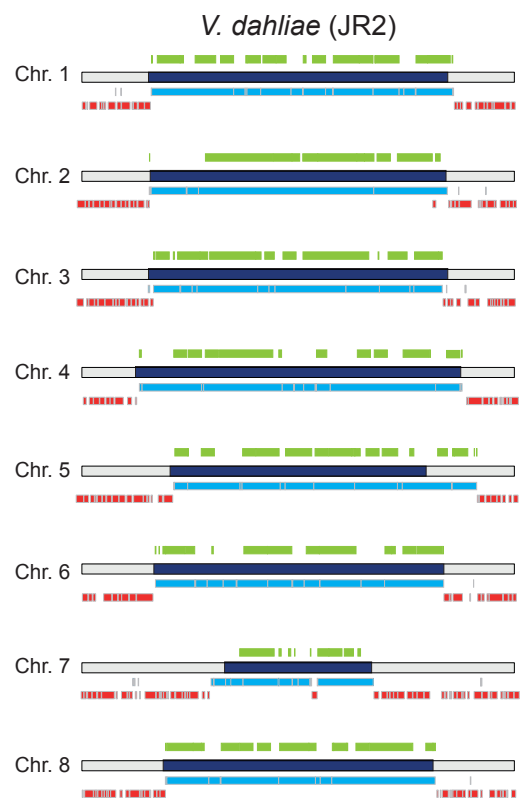

C

Cen

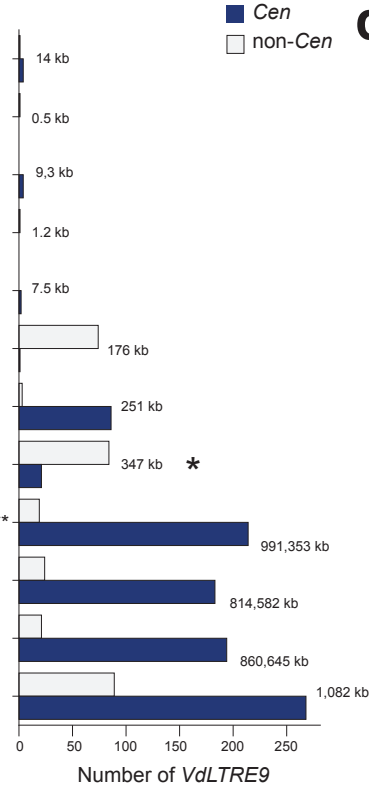

Centromere length $(\mathrm{kb})$
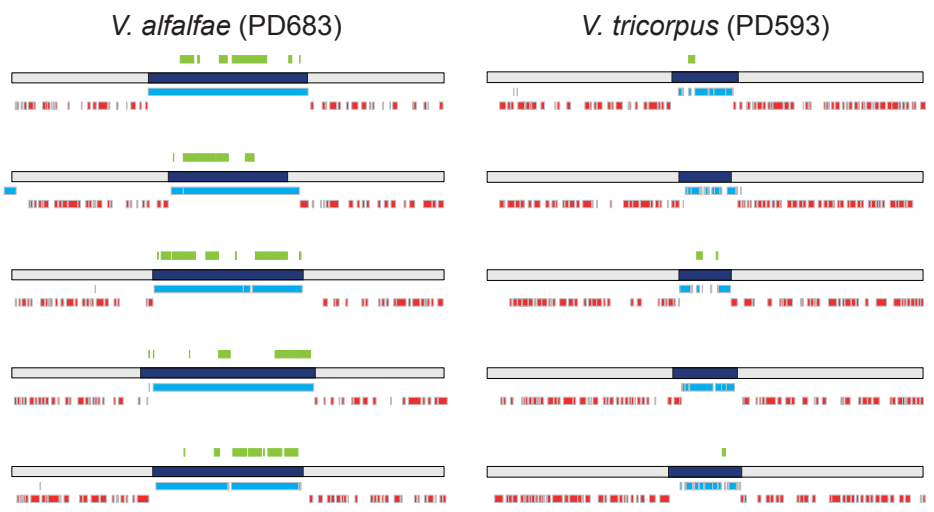

$-1$

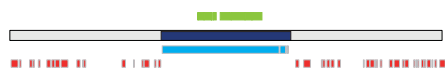

$1+\frac{1}{110}$

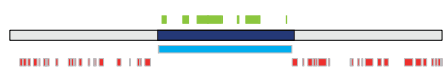

-

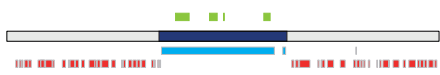

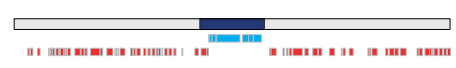
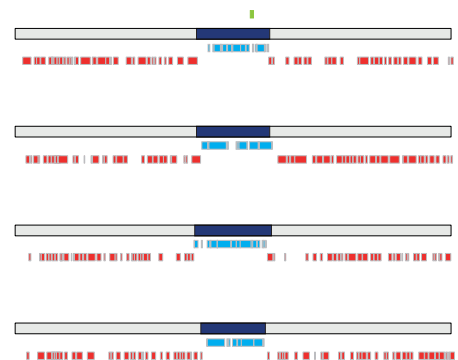

VdLTRE9

Repeats

Genes 
b Centromere-associated rearrangements

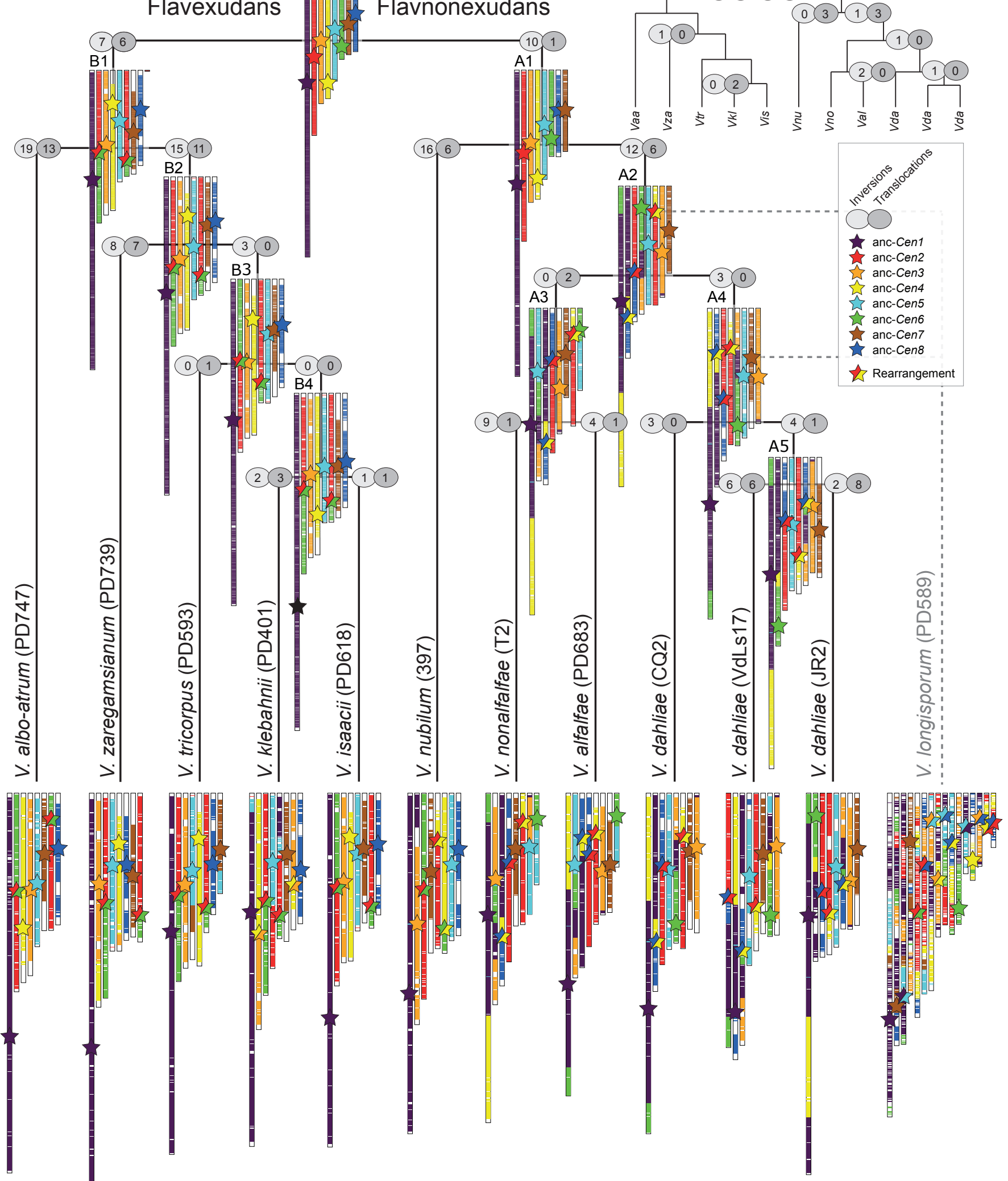


bioRxiv preprint doi: https://doi.org/10.1101/2020.06.29.179234; this version posted June 30, 2020. The copyright holder for this preprint (which was not certified by peer review) is the author/funder, who has granted bioRxiv a license to display the preprint in perpetuity. It is made

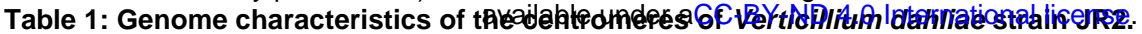

CenH3

AT-rich

AT-content (\%) ${ }^{3}$

Repetitive elements

\begin{tabular}{|c|c|c|c|c|c|c|c|c|}
\hline Chr. & Locus & CenH3 position (bp) & CenH3 length (bp) & Position (kb) ${ }^{2}$ & Chr. & Cen. & \# Repeats (\%) & \# VdLTRE9 (\%) \\
\hline 1 & CEN1 & $2,920,143-3,094,179$ & 174,037 & $2,919-3,094$ & 45.7 & 77.1 & $50(99.8)$ & $27(70.4)$ \\
\hline 2 & CEN2 & $520,698-672,281$ & 151,584 & $516-672$ & 46.3 & 77.8 & $43(99.7)$ & $26(83.0)$ \\
\hline 3 & CEN3 & $2,374,294-2,541,026$ & 166,733 & $2,375-2,542$ & 45.8 & 77.3 & $47(99.8)$ & $31(80.5)$ \\
\hline 4 & CEN4 & $2,884,316-3,071,412$ & 187,097 & $2,885-3,072$ & 46.2 & 75.4 & $54(99.5)$ & $24(53.8)$ \\
\hline 5 & CEN5 & $1,868,317-2,043,260$ & 174,944 & $1,868-2,044$ & 46.7 & 73.9 & $58(99.5)$ & $25(63.1)$ \\
\hline 6 & CEN6 & $2,166,972-2,333,060$ & 166,089 & $2,167-2,334$ & 46.4 & 75.2 & $48(100)$ & $31(62.6)$ \\
\hline 7 & CEN7 & $1,944,367-2,038,091$ & 93,725 & $1,945-2,038$ & 44.7 & 76.5 & $32(95.8)$ & $14(47.8)$ \\
\hline 8 & CEN8 & $1,406,398-1,561,664$ & 155,267 & $1,406-1,562$ & 47.7 & 77.0 & $37(100)$ & $26(73.9)$ \\
\hline
\end{tabular}

1: position of CenH3-enriched domains; enriched domains within $10 \mathrm{~kb}$ have been merged

2: position of AT-rich domains; AT-rich domains with $20 \mathrm{~kb}$ have been merged

3 : average AT-content of $1 \mathrm{~kb}$ windows of the entire chromosome and the AT-rich domain

${ }^{4}$ : percentage of centromeric region covered 